\title{
Scaling and optimization of the radiation temperature in dynamic hohlraums
}

\author{
S. A. Slutz, M. R. Douglas, J. S. Lash, R. A. Vesey, G. A. Chandler, T. J. Nash, and M. S. \\ Derzon
}

Sandia National Laboratories, Albuquerque, N. M. 87185-1186

\section{abstract}

We have constructed a quasi-analytic model of the dynamic hohlraum. Solutions only require a numerical root solve, which can be done very quickly. Results of the model are compared to both experiments and full numerical simulations with good agreement. The computational simplicity of the model allows one to find the behavior of the hohlraum temperature as a function the various parameters of the system and thus find optimum parameters as a function of the driving current. The model is used to investigate the benefits of ablative standoff and axial convergence.

PACS 52.55.Ez, 52.50.Lp, 52.25.Nr 


\section{Introduction}

Pulsed power is a robust and inexpensive technology for obtaining high electrical energy densities, which can be efficiently converted into thermal radiation by $z$ pinches. The use of wire arrays, with a large number of wires, has resulted in remarkable improvements in radiated power from z-pinches. Recently ${ }^{1}, 2 \mathrm{MJ}$ of thermal $\mathrm{x}$-rays have been generated by a $\mathrm{z}$-pinch with an efficiency greater than $15 \%$ and an $x$-ray power exceeding $200 \mathrm{TW}$. This thermal $\mathrm{x}$-ray power has been used to drive a hohlraum ${ }^{2}$ to a temperature greater than $145 \mathrm{eV}$. A promising inertial fusion scenario ${ }^{3}$ is to use two $z$-pinches to drive an external hohlraum containing a fusion capsule as shown in Fig. 1. Calculations ${ }^{3}$ indicate that high yields (400-1200 MJ) can be obtained with $16 \mathrm{MJ}$ of $\mathrm{x}$-ray energy provided by the two pinches. A large fraction of the $\mathrm{x}$-ray energy is absorbed by the hohlraum walls of the primary and secondary hohlraums, which have

$\therefore$ an area of approximately $30 \mathrm{~cm}^{2}$. $\because$

The dynamic hohlraum ${ }^{4}$ is an alternative approach to generating thermal radiation to drive a capsule, which should require less total energy. In this approach, a z-pinch plasma is imploded onto a "convertor", which surrounds the capsule as depicted schematically in Fig 2. In this figure, the $z$-pinch plasma is initially formed at $\mathrm{r}_{\mathrm{z} 0}$ and is imploded onto the convertor, which initially occupies the region $\mathrm{r}_{\mathrm{ci}}<\mathrm{r}<\mathrm{r}_{\mathrm{co}}$. The z-pinch plasma can be generated from a wire array or a solid liner. At the low mass (few $\mathrm{mg}$ ) of the present $\mathrm{z}$ pinches, wires have been the most successful. This is because such low mass liners cannot be constructed without wrinkles, which seed the Rayleigh-Taylor (RT) instability. To maintain a constant implosion time, the mass of the z-pinch increases with driving current, so liners may become practical at the higher currents needed to drive fusion capsules. When the z-pinch plasma first strikes the convertor it is in the region $r_{c o}<r<r_{z o}$. The radius of interface between the $z$-pinch plasma and the convertor, $r_{z i}$, is initially at $r_{c o}$ and decreases with time. Two shock waves are formed at $r_{c o}$, one propagates into the $z$-pinch plasma and the other propagates inward into the convertor. These shock waves heat both regions, which then emit thermal radiation. A material of low opacity (typically a plastic foam) is chosen for the convertor so that radiation can easily flow inward to heat an ICF capsule. The z-pinch plasma is composed of a material with high opacity (tungsten is commonly used) to minimize the outward flow of radiation and thus achieve max- 


\section{DISCLAIMER}

This report was prepared as an account of work sponsored by an agency of the United States Government. Neither the United States Government nor any agency thereof, nor any of their employees, make any warranty, express or implied, or assumes any legal liability or responsibility for the accuracy, completeness, or usefulness of any information, apparatus, product, or process disclosed, or represents that its use would not infringe privately owned rights. Reference herein to any specific commercial product, process, or service by trade name, trademark, manufacturer, or otherwise does not necessarily constitute or imply its endorsement, recommendation, or favoring by the United States Government or any agency thereof. The views and opinions of authors expressed herein do not necessarily state or reflect those of the United States Government or any agency thereof. 
. 


\section{DISCLAIMER}

Portions of this document may be illegible in electronic image products. Images are produced from the best available original document. 
imal radiation temperatures. The z-pinch plasma forms the walls of a hohlraum with much smaller dimensions (several $\mathrm{mm}$ ) than the walls of the double-ended $\mathrm{z}$-pinch driven hohlraum $(\sim 2 \mathrm{~cm})$. Thus we expect the dynamic hohlraum to have less radiation loss and to generate higher radiation temperatures than would be obtained using the double-ended $z$-pinch driven hohlraum with the same total energy. Indeed numerical simulations ${ }^{5}$ indicate that a dynamic hohlraum could drive a high yield capsule $(500 \mathrm{MJ})$ with approximately $12 \mathrm{MJ}$ of kinetic energy delivered to the z-pinch. Furthermore, the performance of the dynamic hohiraum depends on the trade-off between a number of factors, with the likelihood that this design has not been fully optimized. Therefore it is valuable to have a model to determine the performance of the dynamic hohlraum as a function of the many parameters that can be varied. $\mathrm{A}$ simple analytic estimate ${ }^{6}$ of the peak radiation temperature based on shock heating has been made. We present a more complete model that includes the effects of radiation loss, the specific heat of the components, and the radiation absorbed by the capsule. We use our model to find optimum parameters as a function of the driving current.

The model also includes the effects of ablative standoff, where incoming shock waves can be isolated from the capsule by the outgoing ram pressure of the ablated material. This "ablative standoff" has been seen in numerical simulations ${ }^{5}$ of high yield dynamic hohlraum driven capsules. We find that ablative standoff is more effective at larger drive currents.

Axial convergence of an initially cylindrical z-pinch will occur if the z-pinch material has an appropriate axial mass profile, i.e. heavier at the midplane and lighter at the ends. We have performed both simplified and detailed numerical simulations of an initially cylindrical z-pinch with a $2 \mathrm{~cm}$ initial radius and $1 \mathrm{~cm}$ initial axial length, that had a mass/length, $\Lambda$, given by the expression

$$
\Lambda=\Lambda_{0}\left(1+a_{2} z^{2}+a_{4} z^{4}\right)
$$

where $a_{2}=-0.06, a_{4}=0.024$, and $\Lambda_{0}=3.75 \mathrm{mg} / \mathrm{cm}$. The results from the simplified model at various times during the implosion are shown in Fig. 3. As can be seen the final shape is nearly spherical. Similar results have been obtained by others ${ }^{7}$. This quasi-spherical z-pinch implo- 
sion should provide better coupling to the spherical inertial fusion capsule. Indeed, our model predicts significantly higher hohlraum temperatures for quasi-spherical z-pinch implosions than purely cylindrical z-pinch implosions. However, as we shall show both analytically and with detailed numerical simulations, the mass profile needed to produce axial convergence also seeds the Rayleigh-Taylor instability. Experiments will be needed to determine the viability of quasi-spherical z-pinch implosions.

This paper is organized as follows. The model of the dynamic hohlraum is presented in the next section. Results are compared to experiments in section III. In section IV, model results are compared to full radiation hydrodynamic simulations to further benchmark the model. In section $\mathrm{V}$, the model is then used to optimize and predict the scaling of dynamic hohlraums. The model predicts that ablative standoff is more effective at high drive currents. The scaling of the hohlraum temperature with the degree of radiation trapping is found to be fairly weak. The model also predicts an increased peak hohlraum temperature of approximately $20 \mathrm{eV}$ for quasi-spherical pinches. However, the mass profiling that produces the quasi-spherical z-pinch is shown to seed the RT instability. Conclusions are given in section VI. The analytic z-pinch model is described in appendix I. The radiation transport through the z-pinch plasma is described in appendix II. Analytic estimates of the seeding and growth of the RT instability by mass profiling to produce a quasi-spherical z-pinch are presented in appendix III. A glossary of symbol definitions is provided in appendix IV.

\section{Dynamic hohlraum model}

\section{A. Analytic z-pinch solutions}

We are interested in modelling both purely cylindrical and axially convergent z-pinch implosions. Analytic solutions for purely cylindrical and axially convergent $z$-pinches are developed in Appendix I. The normalized radius and current for a cylindrical pinch are plotted as a function of time in Fig. 4. The initial mass of the z-pinch is found to be 


$$
M_{Z}=\frac{(3-2 \alpha)^{2-\alpha}}{12(2-2 \alpha)^{1-\alpha}}\left(\frac{\mu_{0}}{4 \pi}\right)\left(\frac{I_{P} t_{r}}{r_{Z 0}}\right)^{2} L_{Z}
$$

where $I_{U}$ is the peak driving current, $t_{T}$ is the risetime, $L_{p}$ is the initial length of the pinch, $r_{z 0}$ is the initial radius of the pinch, and $\alpha$ is a parameter which determines the degree of axial convergence. Cylindrical pinches correspond to $\alpha=0$, while the axially converging pinch shown in Fig 3 corresponds to $\alpha=1 / 3$.

The kinetic energy of the pinch is found to be

$$
E_{K}=\frac{2(3-2 \alpha)^{\frac{3}{2}-\alpha}}{3(2-2 \alpha)^{1-\alpha}} L_{Z}\left(\frac{\mu_{0}}{4 \pi}\right) I_{P}^{2}\left(1-\frac{r}{r_{z 0}}\right)^{3 / 2}
$$

where $r$ is the radius of the pinch. A comparison between eq. (3) $(\alpha=0)$ with a cylindrical numerical solution, which includes inductive feed back on the current is shown in Fig. 5. The agreement is excellent for convergence ratios expected in dynamic hohlraums, i.e. less than 5 . The analytic solution facilitates optimization, which involves finding many solutions.

\section{B. Capsule implosion times}

The collision between the z-pinch plasma and the convertor generates shocks which propagate through both the $\mathrm{z}$-pinch plasma and the convertor. During the propagation of these shocks, heat is produced with a subsequent rise in the hohlraum temperature. For thin convertors the shock will pass completely through the convertor before the fusion capsule fully implodes. This will also be true for the relatively thin z-pinch plasma. The convertor and the zpinch plasma will then be at high pressure and thus difficult to compress futher, so no significant heating will occur after these initial shocks have passed through the convertor and the zpinch plasma. Thus the hohlraum temperature will reach its peak when the shock has just passed through the convertor and then the hohlraum temperature will decrease slowly as the convertor continues to collapse inward. We shall approximate this behavior by assuming the hohlraum temperature rises linearly with time up to its peak value, $T_{H P}$ in a risetime, $\tau_{\Gamma}$ equal to the time it takes for the stagnation shock to pass through the convertor and then stays constant until the capsule implodes. The initial radius of the convertor is chosen so that the 
imploding material will not directly interact with the fusion capsule for the time it takes the capsule to implode, $\tau_{\text {cap }}$

The high yield design ${ }^{5}$ uses a foam convertor that extends to the axis, i.e. the capsule is imbedded in the foam. This design results in rising hohlraum temperature during the capsule implosion. Such convertors also couple more of the stagnation energy into the convertor and the relatively slow rise of the hohlraum temperature yields a reasonably isentropic implosion of the fusion capsule. For simplicity, we assume that the rise is linear with time, i.e. $T_{H}(t)=$ $\mathrm{tT}_{\mathrm{HP}} / \tau_{\text {cap }}$. Again the initial outer radius of the foam must be chosen so that the $z$-pinch material does not stagnate on the capsule before it implodes, since this would destroy the capsule symmetry.

We shall now estimate the capsule implosion time. Newton's equation for the radius of a capsule during implosion can be written

$$
m_{\text {cap }}\left(r_{c}\right) \frac{d^{2} r_{c}}{d t^{2}}=4 \pi r_{c}^{2} P_{a b l} \text {, }
$$

where $r_{c}$ is the radius of the capsule as a function of time, $m_{\text {cap }}\left(r_{c}\right)$ is the unablated capsule mass and the ablation pressure depends on the hohlraum temperature. The ablation pressure at the surface of an inertial fusion capsule is approximately ${ }^{8}$

$$
\mathrm{P}_{\mathrm{abl}}=3.3 \times 10^{4} \mathrm{~T}_{\mathrm{H}}^{3.5}
$$

where $\mathrm{T}_{\mathrm{H}}$ is the radiation temperature in units of eV and the ablation pressure is in Pascals. For thin convertors, we shall assume constant hohlraum temperature during the capsule implosion. Since the mass of the capsule decreases due to ablation, we assume the convenient form $\mathrm{m}_{\text {cap }}(\mathrm{r})=\mathrm{M}_{\text {cap }} \mathrm{r} / \mathrm{r}_{\text {cap }}$, where $\mathrm{M}_{\text {cap }}$ is the initial capsule mass and $r_{\text {cap }}$ is the initial capsule radius. Using this form eq. (4) can be solved analytically. The result is

$$
\tau_{\text {cap }}=\frac{\pi r_{\text {cap }}}{2 v_{f}}
$$


The final implosion velocity, $\mathrm{v}_{\mathrm{f}}$, of an inertial fusion capsule must be approximately $35 \mathrm{~cm} / \mu \mathrm{s}$ to obtain ignition ${ }^{8}$. For solid low density convertors, we assume a linear rise in the hohlraum temperature up until the capsule implosion time, which we shall also refer to as "bang time". Equation (4) can be solved assuming a linear rise in the hohlraum temperature. The result is

$$
\tau_{\text {cap }}=\frac{5.5 \mathrm{r}_{\text {cap }}}{\mathrm{v}_{\mathrm{f}}}
$$

\section{Geometric considerations: radiation symmetry and standoff}

Numerical simulations indicate that inertial fusion capsules require a radiation asymmetry of $1 \%$ or less ${ }^{8}$. Thus there must be sufficient space between the z-pinch plasma and the capsule so that the radiation can transport freely around the capsule to provide a symmetric radiation drive. This condition is satisfied if the $\mathrm{z}$-pinch/convertor interface radius is larger than some minimum radius during the capsule implosion. We define a symmetry parameter

$$
F_{s}=\frac{r_{z i b}}{r_{\text {cap }}}
$$

where $r_{\text {zib }}$ is the inner radius of the z-pinch plasma at capsule bang time. Large values of $F_{s}$ will allow a high degree of radiation smoothing, but will also result in relatively low hohlraum temperatures. Thus it is important to have at least an estimate of the value of $F_{s}$. Note that $F_{s}$ can be less than unity because the capsule is imploding as the inner radius of the z-plasma decreases.

Lasnex ${ }^{9}$ simulations show that the Rayleigh-Taylor (RT) instability modulates the $z$ plasma thickness with a wavelength of approximately $1 \mathrm{~mm}$, see Fig. 6 . This simulation indicates that the radiation source has a variation, $\delta \mathrm{T}^{4} \mathrm{~T}^{4} \sim 30 \%$ for a wavelength of $1 \mathrm{~mm}$. Note however, that the amplitude is not the same for each bubble and spike. This indicates the presence of longer wavelengths.

The radiation flux incident on the capsule surface can be much more uniform than the radiation source at the z-pinch plasma, which acts as the hohlraum wall. This is because radiation incident on each point of the capsule is a solid angle weighted integral over the visible hohl- 
raum wall. We have performed these integrals in a "view factor" code. The hohlraum wall and the radiation source were assumed to be a cylinder with a radius of $R_{\text {hohl }}$ and length $L_{h o h l}$, with a constant ratio $L_{\text {hohl }} / R_{\text {hohl }}=4$. The capsule was assumed to have a constant radius equal to the initial capsule radius, $r_{\text {cap }}$. The radiation flux emitted from the hohlraum wall was given a sinusoidal axial variation with a fixed wavelength to simulate the effect of the RT instability. The albedo of the walls was set to zero (due to present limitations of the view factor code), which results in an underestimate of the effect of radiation smoothing. Calculations were performed for several values of the ratio $R_{\text {hohl }} / r_{\mathrm{c}}$, and the source wavelength, $\lambda$, for fixed relative variation of the source amplitude, $\delta \mathrm{F} / \mathrm{F}_{0}$, where $\mathrm{F}_{0}$ is the source amplitude without any variations due to $\mathrm{RT}$ and $r_{\mathrm{c}}$ is the capsule radius at the time of interest during its implosion. Calculations with $\delta \mathrm{F} / \mathrm{F}_{0}=0$, result in an $34 \%$ variation of the radiation flux at the capsule from equator to pole. This unperturbed equator to pole variation results from the end walls (the electrodes), which are not radiation sources. This variation is reduced as the electrode albedo is increased to reasonable values for gold ( $\alpha=0.8$ gives a $6 \%$ variation). The remaining variation, which should be the same on each shot, can be compensated for by design, e.g. varying the thickness and/or radius of the convertor as a function of axial position. In contrast, the variation caused by the RT instability will be different on each shot. To assess the effect of the RT instability, we subtracted this unperturbed variation from calculations with the sinusoidal perturbations and expanded the results into Legendre modes. Since fusion capsules require a time averaged radiation asymmetry of $1 \%$ or better (summed over all modes), we inverted our results to find the required source amplitude to obtain $1 \%$ variation on the capsule, $\delta \mathrm{F}_{1}$, as a function of the ratio $\frac{R_{\text {hohl }}}{r_{c}}$. The results are plotted in Fig. 7 for three different wavelengths, $\lambda=1,2$, and $4 \mathrm{~mm}$. As $r_{c}$
can be seen from the figure, $\delta F_{1}$ is a strong function of both $\frac{R_{\text {hohl }}}{r_{c}}$ and the wavelength. The results indicate that the $\lambda=1 \mathrm{~mm}$ source variations of $30 \%$ seen in the Lasnex simulations should be smoothed to approximately $1 \%$ at the capsule for the ratio $R_{\text {hohl }} / r_{c}=2.0$. Adequate radiation symmetry will be achieved if the ratio of the inner radius of the z-plasma over the capsule radius is never smaller than approximately 2 . We have solved eq. (4) to determine the capsule radius as a function of time. Comparing this to the radius of the $\mathrm{z}$-pinch as a function of time we find that a ratio $F_{s}>0.5$ is usually large enough to insure this condition is satisfied. 
Note that the Lasnex simulations show that the RT introduces some structure at wavelengths longer than $1 \mathrm{~mm}$. If such longer wavelength modes have sufficient amplitude in the experiments, larger values of $F_{s}$ may be needed to obtain adequate radiation symmetry.

We wish to calculate the initial radius of the convertor needed to isolate the capsule from the imploding plasmas. The velocity will be affected by the collision between the z-pinch and the convertor and also the magnetic acceleration. Using conservation of momentum the average velocity of the inner radius of the z-plasma for a thin convertor is found to be approximately

$$
\overline{\mathrm{v}}_{\mathrm{z}}=\frac{\mathrm{M}_{\mathrm{Z}}}{\left(\mathrm{M}_{\mathrm{Z}}+\mathrm{M}_{\mathrm{C}}\right)} \frac{\left(\mathrm{v}_{\mathrm{z}}\left(\mathrm{r}_{\mathrm{zib}}\right)+\mathrm{v}_{\mathrm{z}}\left(\mathrm{r}_{\mathrm{co}}\right)\right)}{2},
$$

where the velocity, $v_{z}$ of the imploding $z$-pinch plasma without a convertor is found from eq. A1.8. The situation is different for a solid low density convertor, where the collision occurs during the entire implosion. Assuming that shock has propagated through all of the convertor by the time the $z$-pinch/convertor radius reaches $\mathrm{r}_{z \mathrm{zib}}$ we obtain the approximate express for the average velocity,

$$
\bar{v}_{z}=\frac{1}{2}\left(v_{z}\left(r_{c o}\right)+\frac{v_{z}\left(r_{z i b}\right) M_{z}}{M_{Z}+M_{C}}\right)
$$

The initial outer radius of the convertor, $r_{c o}$, can be chosen so that the inner radius of the $z$ plasma just equals $r_{z i b}$ at the bang time of the capsule. We then find the initial outer radius of the convertor to be

$$
\mathrm{r}_{\text {co }} \approx \mathrm{r}_{\mathrm{zib}}+\overline{\mathrm{v}}_{\mathrm{z}} \tau_{\text {cap }}
$$

For solid low density convertors, $r_{\mathrm{ci}}=0$, therefore a shock wave can arrive at the capsule before the interface reaches $r_{z i b}$ This is a potential problem, because the shock wave could disrupt the spherical symmetry of the fusion capsule. However, under appropriate conditions, the ablation pressure is larger than the shock pressure and the shock will remain isolated from the capsule. We call this ablative standoff. The pressure of the shock wave is given by the expression ${ }^{10}$ 


$$
P_{s h}=\frac{2 \rho_{c} v_{s}^{2}}{\gamma+1}
$$

where $\rho_{\mathrm{c}}$ is the initial convertor density. The z-pinch plasma acts as a piston driving a shock wave into the convertor. The shock wave will move ahead of the interface. Assuming an ideal gas with $\gamma=5 / 3$, the shock will move at $4 / 3$ times the velocity of the interface. In addition the shock will be accelerated by cylindrical or spherical convergence. Analytic solutions ${ }^{11}$ show that the shock velocity is proportional to $r^{-\mu}$ where $\mu=0.226$ for cylindrical and $\mu=0.45$ for spherical convergence. Thus the velocity of the shock, when it arrives at the capsule, is approximately

$$
\mathrm{v}_{\mathrm{s}}=\frac{4 \overline{\mathrm{v}}_{z}}{3}\left(\frac{\mathrm{r}_{\mathrm{co}}}{\mathrm{r}_{\mathrm{cap}}}\right)^{\mu}
$$

The ablation pressure generates an outward flow of material which has a ram pressure given by

$$
\mathrm{P}_{\mathrm{ram}}=\left(\frac{\mathrm{r}_{\mathrm{cap}}}{\mathrm{r}}\right)^{2} \mathrm{P}_{\mathrm{abl}}
$$

where $r_{\text {cap }}$ is the initial capsule radius and $P_{a b l}$ is found from eq. (5). Note that the capsule will not have imploded much at the time that the first shock arrives at the capsule. Setting $\mathrm{P}_{\mathrm{ram}}=$ $\mathrm{P}_{\mathrm{sh}}$, we can solve for, $\mathrm{r}_{\mathrm{ab}} / \mathrm{r}_{\mathrm{cap}}$, the ablative standoff radius normalized to the capsule radius. The result is

$$
\frac{r_{a b}}{r_{c a p}}=\frac{210}{v_{s} \sqrt{\rho_{c}}} T_{H}^{1.75}\left(t_{1}\right)
$$

where

$$
\mathrm{T}_{\mathrm{H}}\left(\mathrm{t}_{1}\right)=\frac{\mathrm{t}_{1} \mathrm{~T}_{\mathrm{HP}}}{\tau_{\text {cap }}} \cong \frac{\mathrm{T}_{\mathrm{H}}}{\beta_{\mathrm{s}}},
$$

is the hohlraum temperature when the shock arrives at the capsule, since the average shock velocity is approximately

$$
\overline{\mathrm{v}}_{\mathrm{s}} \cong \frac{4(1+\mu)}{3} \overline{\mathrm{v}}_{\mathrm{z}}=\beta_{\mathrm{s}} \overline{\mathrm{v}}_{\mathrm{z}}
$$


where $\beta=1.63$ for a cylindrical pinch and $\beta=1.93$ for a quasi-spherical pinch.

If $r_{a b}>r_{c a p}$ the incoming shock will be reflected outward. This reflected shock will typically reach the interface before $\tau_{\text {cap }}$, and slow incoming z-pinch plasma. Similarly a shock will be reflected from the axis for $|z|>r_{\text {cap }}$. This reflected shock will also propagate outward and slow the incoming z-pinch plasma, but because of the time delay the $\mathrm{z}$-pinch plasma will be distorted into a non-cylindrical shape. For simplicity we assume the z-pinch remains cylindrical for our model calculations.

On the other hand, if the shock pressure is larger than the ablation pressure of the capsule, the spherical symmetry of the capsule will be destroyed, unless the initial outer radius of the convertor is large enough so that the capsule is isolated from the shock. This will be true if the initial outer radius of the convertor is approximately

$$
r_{\text {co }} \approx r_{\text {cap }}+\bar{v}_{s} \tau_{\text {cap }}
$$

Note that this is considerably larger than the value given by eq. (11). The implies that the final radius of the interface will be larger by the approximate factor $3 / F_{s}$. Therefore, a solid convertor will not work as well as a thin hollow convertor if ablative standoff is not operative.

There is some pinch length for a given capsule and accelerator that will generate the highest hohlraum temperature. However, our model is not able to find this optimum since the analytic solution for the pinch dynamics does not include the feed back between pinch length and driving current. Therefore, we have simply fixed the length of the pinch at roughly 5 times the capsule radius, which is the ratio chosen in the high yield design ${ }^{5}$. Lasnex calculations indicate that the hohlraum temperature is a weak function of this ratio near this value.

\section{Energy balance and radiation transport}

The z-pinch plasma of mass $\mathrm{M}_{Z}$ collides with the convertor of mass $\mathrm{M}_{\mathrm{C}}$. For thin convertors, shock waves will travel completely through both of these regions, bringing them at rest with respect to each other. This is essentially a simple inelastic collision so the energy available to heal the materials and generate radiation is approximately 


$$
E_{A}=E_{k}\left(\frac{M_{C}}{M_{C}+M_{Z}}\right)
$$

One might at first think that we should also use $r_{z i b}$ to compute the kinetic energy, since work is being done by the magnetic field on the z-pinch plasma after first strike. However, once the initial shock waves have propagated through both the z-pinch plasma and the convertor the materials are at high pressure and will be difficult to compress further. Thus most of this work will go into kinetic energy, which can not be converted into radiation until these plasmas stagnate on axis. Thus for thin convertors we shall use $r_{c o}$ to calculate the available kinetic energy from eq. (3).

The situation is more complicated for solid low density convertors, since the shock can transit the convertor several times. This is because the shock can be reflected from either the capsule ablation surface or the axis. For simplicity we will ignore the presence of the capsule. The shock will reflect off the axis and return to the inner radius of the z-pinch plasma, when it is at the position

$$
r_{2}=\frac{\beta_{s}-1}{\beta_{s}+1} r_{c o}
$$

At this time the convertor material has been shocked twice. The inward propagating shock drove the convertor material inward at approximately $3 \bar{v}_{z} / 4$. Using the shock relations one can show that the outward shock will bring the velocity of the convertor material back to zero and the the energy that remains in the z-pinch plasma is approximately $\left(\frac{M_{Z}}{M_{Z}+M_{C}}\right)^{2} E_{k}$. When the inner radius of the z-pinch plasma reaches $r_{z i b}$ the available energy is then given by the expression

$$
E_{A}=\left[1-\left(\frac{M_{Z}}{M_{Z}+M_{C}}\right)^{N_{s}}\right] E_{K}
$$

where the shocks have crossed the convertor $\mathrm{N}_{\mathrm{s}}$ times with 


$$
\mathrm{N}_{\mathrm{S}}=\frac{2 \ln \left(\frac{\mathrm{r}_{\mathrm{zib}}}{\mathrm{r}_{\mathrm{co}}}\right)}{\ln \left(\frac{\beta_{\mathrm{s}}-1}{\beta_{\mathrm{s}}+1}\right)} .
$$

This expression yields an available energy significantly larger than is obtained for the annular convertors. As an example, assuming $M_{C}=M_{Z}, E_{A} / E_{K}=1 / 2$ for a thin convertor, but $E_{A} / E_{K}$ can be greater than 0.8 for a solid convertor.

A portion of the available energy is deposited in the convertor with the rest going into the z-pinch plasma. We estimate these fractions from the following argument. In the center of mass frame for the collision, two stagnation shocks will propagate away from the initial point of contact. The material between these shocks will have little relative motion, so the pressure must be nearly constant in these regions. Since pressure has units of energy density, the fraction of the energy that goes into the z-pinch plasma will be approximately

$$
f_{z}-\frac{d_{z}}{d_{C}+d_{z}}
$$

where $d_{C}$, is the thickness of the convertor and $d_{Z}$ is the thickness of the $z$-pinch plasma at first contact. A portion of this energy will go to internal energy, while the rest will go into radiation, i.e.

$$
E_{A}=E_{I N T}+E_{R A D}
$$

At any point in time, the radiation temperature will be nearly spatially constant within the convertor and the hohlraum, since we have chosen a low opacity material for the convertor. We define the hohlraum temperature, $\mathrm{T}_{\mathrm{H}}(\mathrm{t})$, to be the radiation temperature within this region. In contrast, the radiation temperature will have significant spatial variation within the z-pinch plasma due to the high opacity of this material. Let $R_{Z H}$ be the ratio of the spatial average of the radiation temperature in the $\mathrm{z}$-pinch plasma as compared to $\mathrm{T}_{\mathrm{H}}$, the hohlraum temperature. To find $\mathrm{R}_{\mathrm{ZH}}$ we need to solve the radiation transport to obtain the radiation temperature profile. Then the total internal energy is given by

$$
E_{I N T}=E_{Z}\left(R_{Z H} T_{H}\right) M_{Z}+E_{C}\left(T_{H}\right) M_{C} \text {, }
$$


where $E_{z}$ and $E_{c}$ are the internal energy functions for the z-pinch plasma material and the convertor material. We approximate these functions with power law fits to data in the Sesame Equation of State package ${ }^{12}$. Assuming tungsten for the z-pinch material we obtain

$$
E_{Z}(T)=7.0 \times 10^{6} T_{e v}^{1.35} \rho^{-0.15} \mathrm{~J} / \mathrm{kg},
$$

where $\mathrm{T}_{\mathrm{ev}}$ is the temperature in units of $\mathrm{eV}$, and $\rho$ is in $\mathrm{g} / \mathrm{cm}^{3}$. Assuming $\mathrm{CH}_{2}$ for the convertor the energy function is

$$
E_{C}(T)=2.4 \times 10^{8} \mathrm{~T}_{\mathrm{ev}}^{0.88} \mathrm{~J} / \mathrm{kg}
$$

The energy that goes in to radiation is composed of three parts

$$
E_{\mathrm{RAD}}=\mathrm{E}_{\text {cap }}+\mathrm{E}_{\mathrm{W}}+\mathrm{E}_{\mathrm{S}} \text {, }
$$

where $E_{c a p}$ is the energy absorbed by the capsule, $E_{w}$ is the energy absorbed by the electrode walls, and $E_{s}$ is the energy radiated from the outer surface of the $z$-pinch plasma. The radiation absorbed by the ICF capsule is given by the expression

$$
E_{c a p}=\left(1-\alpha_{c}\right) 4 \pi \sigma \int_{0}^{\tau_{b}} r_{c}^{2}(t) T_{H}^{4}(t) d t=\left(1-\alpha_{c}\right) 4 \pi \sigma r_{c a p}^{2} T_{H P}^{4} \tau_{r c}
$$

where $\tau_{b}$ is the capsule bang time, $\alpha_{c}$ is the effective albedo of the capsule, $r_{c}(t)$ is the capsule radius as a function of time, $T_{H}(t)$ is the hohlraum temperature as a function of time, $r_{c a p}$ is the initial capsule radius, and $\tau_{\mathrm{rc}}$ is the effective capsule radiation absorption time as defined by second equality. For solid convertors, $\tau_{b}=\tau_{c a p}$, but for annular convertors, $\tau_{b}=\tau_{c a p}+\tau_{r}$ Since the outer material of the capsule is chosen to effectively absorb radiation the albedo is low. We use the value of 0.3 , which is consistent with typical plastic ablator materials. A portion of the electrode walls will be exposed to the hohlraum radiation $\left(r<r_{z i}\right)$ as shown in Fig. 2. The radiation lost to the electrodes is given by

$$
\mathrm{I}_{\mathrm{w}}=\left(1-\alpha_{\mathrm{w}}\right) 2 \pi \sigma \int_{0}^{\tau} \mathrm{r}_{\mathrm{zi}}^{2}(\mathrm{t}) \mathrm{T}_{\mathrm{H}}^{4}(\mathrm{t}) \mathrm{dt}=\left(1-\alpha_{\mathrm{w}}\right) 2 \pi \mathrm{r}_{\mathrm{co}}^{2} \sigma \mathrm{T}_{\mathrm{H}}^{4}(\tau) \tau_{\mathrm{rw}}
$$

where $\alpha_{\mathrm{w}}=0.8$, is the albedo of the wall, $\mathrm{r}_{\mathrm{zi}}(\mathrm{t})$ is the radius of the inner surface of the $\mathrm{z}$-pinch plasma and the convertor as a function of time, and $r_{c o}$ is the initial outer radius of the conver- 
tor. Time dependent dynamic hohlraum radiation temperatures have been measured on the $Z$ accelerator. To compare our model with these experiments we parameterize the model calculations with respect to $\tau$ to yield a crude time dependence. Note that this time dependence is not fully self consistent since we assumed the form of $\mathrm{T}_{\mathrm{H}}(\mathrm{t})$ within the integral. For the rest of our results we shall set $\tau=\tau_{b}$ and only calculate the peak hohlraum temperature, $T_{H P}=T_{H}\left(\tau_{b}\right)$.

The radiation lost from the outer surface of the z-pinch plasma, $E_{S}$, is given by the expression

$$
\Sigma_{S}=4 \pi L_{Z} \sigma \int_{0}^{\tau} T_{0}^{4}(t) r_{z o}(t) d t=4 \pi r_{z o b} L_{Z} \sigma T_{0}^{4}(\tau) \tau_{r},
$$

where $r_{z o}(t)$ is the outer radius of the $z$-pinch plasma from the time of first strike and $T_{o}(t)$ is the outer radiation temperature of the $z$-pinch plasma. Note the solution for the flux from a plane photosphere ${ }^{10}$ is $F=2 \sigma T_{o b}^{4}$, where $T_{o b}=T_{o}\left(\tau_{b}\right)$. The factor of two is due to the increase in temperature going inward from the outer boundary. This factor of two is included in eq. (31). The radiation temperature at the outer boundary of the z-pinch plasma can be determined as a function of the radiation temperature within the hohlraum by solving the radiation transport through the $z$-pinch material $\left(r_{\mathrm{zi}}<r<r_{\mathrm{zo}}\right)$. Since the $\mathrm{z}$-pinch material is highly opaque we use the radiation diffusion approximation. The details are given in Appendix II.

\section{E. The Rayleigh-Taylor instability}

The z-pinch plasma will suffer disruption from the Rayleigh-Taylor (RT) instability as the plasma is imploded. This will decrease the effective opacity of this region, since there will be regions that are much thinner than the average, see Fig. $6 \mathrm{a}$. The mode ${ }^{13}$ of Desjarlais and Marder indicates that approximately $90 \%$ of the $z$-pinch plasma material will be in the Taylor spikes. We shall use the shape shown in Fig. 8 where the curve is assumed to be quadratic. Thus the overall thickness is $\Gamma=\Gamma_{\min }+a^{2}$ where the constant can be determined from the ratio of the spike/bubble mass, $R$. The result is $\Gamma=\Gamma_{\min }\left(1+3 R x^{2}\right)$, where $x=\frac{2 z}{\lambda_{R T}}$. Since radiation transport depends on the Rosseland opacity we want to find the mean 


$$
\frac{1}{\Gamma_{\text {Ross }}}=\frac{1}{\Gamma_{\min }} \int_{0}^{1} \frac{\mathrm{dx}}{1+3 \mathrm{Rx}^{2}}=\frac{\operatorname{atan} \sqrt{3 \mathrm{R}}}{\Gamma_{\min } \sqrt{3 \mathrm{R}}}
$$

For $R=9$ we obtain $\Gamma_{\text {Ross }}=3.76 \Gamma_{\min }$. Since $90 \%$ of the mass is in the spikes for $R=9$, the thickness would be $10 \Gamma_{\min }$ without instabilities. Thus we shall multiply the opacity of the z-pinch plasma by the factor, $F_{R T}=0.376$, to take into account the reduced radiation trapping that results from the RT instability. As we show in section $\mathrm{V}$, this leads to a reduction in the peak hohlraum temperature by approximately $20 \mathrm{eV}$.

\section{F. Numerical procedure}

The numerical procedure for using the model equations presented in this section to obtain the peak hohlraum temperature is outlined in this subsection. The first step is to define various physical quantities that define a specific dynamic hohlraum setup. These parameters include; $\mathrm{I}_{\mathrm{p}}$, the peak current (typically 20-60 MA); $t_{\mathrm{t}}$ the rise time to peak current (typically $100 \mathrm{~ns}$ ); $\alpha_{c}$ and $\alpha_{w}$, the capsule and electrode wall albedos; $F_{s}$, symmetry factor $\left(r_{z i b} / r_{c a p}\right) ; r_{c a p}$, the initial capsule radius; $r_{z 0}$, the initial radius of the $z$-pinch; $L_{z}$, the length of the pinch (typically $5 r_{\text {cap }}$ ); $v_{f}$, the final capsule implosion velocity (typically $35 \mathrm{~cm} / \mu \mathrm{s}$ ); $d_{z}$, the thickness of the z-pinch plasma when it first strikes the convertor $(\sim 2 \mathrm{~mm}) ; \mathrm{M}_{C} / \mathrm{M}_{Z}$, the ratio of the convertor over the z-pinch masses. The capsule implosion time is determined by eq (6) for a thin annular convertor or eq. (7) for a solid convertor. The radius of the inner surface of the z-pinch plasma, at capsule bang time, is determined from $F_{s}$ and the capsule radius. The outer radius of the $z$-pinch plasma at capsule bang time, $r_{z o b}$, is given by the expression $r_{z o b}=r_{z i b}+d_{z} / 4$ where we have assumed the z-pinch plasma has been compressed by a factor of four due to the strong shock produced by the collision with the convertor. The mass of the z-pinch plasma is determined from eq A1.6, where $\alpha=0$ for a cylindrical pinch and $\alpha=1 / 3$ for a quasi-spherical pinch. The convertor mass is then determined by the ratio $M_{C} / M_{Z}$. The average velocity of the inner surface of the z-pinch plasma, $\bar{v}_{z}$, can be calculated from eqs. (9)-(11), and A1.8. This also yields a value for the initial outer radius of the convertor, $r_{c o}$. The average shock velocity is then found from eq. (17). 
At this point we make an initial guess at the peak hohlraum temperature, $\mathrm{T}_{\mathrm{HP}}$ Using this temperature we will define a function, which will be zero only if the correct temperature was chosen. The solution to our problem will then be to find the root of this function.

The kinetic energy of the pinch, $E_{K}$, is found from eq. A1.7. The energy available to heat the materials, $E_{A}$, is found from eq. (19) for thin annular convertors. Otherwise for solid convertors, eqs. (21) and (22) must be used to find the available energy and we must solve for $r_{a b} / r_{c}$, using eqs. (13) and (15). The available energy will be divided into the internal energy of the convertor and z-pinch materials and to radiation. The internal energy is determined by eq. (25). This result depends on the ratio, $R_{Z H}$, of the average radiation temperature within the $z$ pinch plasma over the peak hohlraum temperature. The temperature profile within the z-pinch plasma is determined from eq. A2.8 (for cylindrical pinches) or from eq. A2.9 (for quasi-spherical pinches), where the opacity is determined by eq. A2.6 multiplied by $F_{R T}$ to account for the decreased mean thickness of the z-pinch plasma which results from the RT instability. The radiation absorbed by the capsule is determined by eq. (29) and the radiation absorbed by the electrode wall is determined by eq. (30). The remaining energy must be radiated from the outer surface of the $z$-pinch plasma. This energy, $E_{S}$, defines the radiation temperature, $T_{0}$, at the outer surface of the z-pinch at bang time through eq. (31), and also the specific energy deposition rate through eqs. (23) and A2.3. Setting $r=r_{z i}$ in eq. A2.8 (cylindrical) or eq A2.9 (quasispherical) we can solve for the hohlraum temperature to obtain a new value, $\tilde{T}_{\mathrm{HP}}$. Consistency requires that we find the root of the equation

$$
f\left(T_{H P}\right)=\tilde{T}_{H P}-T_{H P},
$$

which we do numerically.

\section{Model results compared to experiments}

A number of experiments have been performed on the $\mathrm{Z}$ accelerator to study the behavior of the dynamic hohlraum ${ }^{14}$. In this section we shall compare our model calculations to some of these dynamic hohlraum experiments. These comparisons are made for the purpose of bench 
marking the model so that we can have some confidence in model calculations using drive currents that are not presently accessible to experiment.

\section{A. Foam convertor experiments}

Two experiments were performed on the $\mathrm{Z}$ accelerator using solid cylindrical convertors composed of low density plastic foam. On shot 291 , the outer foam radius was $4 \mathrm{~mm}$ with a density of $6 \mathrm{mg} / \mathrm{cc}$. On shot 297 the radius of the convertor was $2.5 \mathrm{~mm}$ and the foam density was $14.3 \mathrm{mg} / \mathrm{cc}$. The mass of the convertors were approximately equal in these two experiments. The rest of the experimental set up-was the same for these two experiments. A current pulse rising to approximately $20 \mathrm{MA}$ in $100 \mathrm{~ns}$ was delivered to a $1 \mathrm{~cm}$ long array of 240 wires at initial radius of $2 \mathrm{~cm}$. An inner array of 120 wires was located at a radius of $1 \mathrm{~cm}$. The presence of the inner "nested" array reduces the effect of the RT instability on the z-pinch implosion $^{15}$. The wires had a diameter of $7.5 \mu \mathrm{m}$ and were composed of tungsten. The total mass of the outer array was $2 \mathrm{mg}$, while the inner array had a mass of $1 \mathrm{mg}$. The radiation temperature was measured by a set of $\mathrm{x}$-ray diodes, bolometers, and time-resolved $\mathrm{x}$-ray pinhole cameras ${ }^{16}$. The hohlraum radiation for shot 291 and 297 are plotted (symbols) as a function of time in Figs. 9. and 10. As can be seen the hohlraum radiation temperature is considerably higher for the smaller radius convertor.

To model these experiments we assume that a single wire array of mass $3 \mathrm{mg}$ at an initial radius of $1.73 \mathrm{~cm}$, which is the mass weighted mean of the nested arrays. The incoming zpinch plasma was assumed to be $2 \mathrm{~mm}$ thick when it first makes contact with the convertor. This thickness is roughly consistent with the radiation pulse widths that have been observed ${ }^{15}$ in nested wire array pinches that are allowed to stagnate on axis. The parameter $\tau$ in eqs. (30) and (31) was used to generate a rough time dependence to compare to the data. These results are also plotted (solid line) in Figs. 9 and 10. As can been seen the agreement between the model and the experiments is quite good. In fact the agreement may seem surprisingly good given the number of approximations that have been made to construct the model. However, one should keep in mind that the radiation temperature is determined by the fourth root of the radiation flux, which is determined by energy balance, and is therefore not very sensitive to errors. 
There are several reasons for the higher temperature in the smaller convertor. At any time after the collision between the $z$-pinch and the convertor, the outer radius of the $z$-pinch plasma is larger for the larger convertor. Thus the outer surface area is larger. Initially the ratio is $4 / 2.5=1.6$, but the ratio increases with time. In addition, the electrode area within the pinch is proportion to the square of the inner radius of the z-pinch plasma. The radiation losses are proportion to these two areas. Furthermore, at any time after first strike, the fraction of the pinch kinetic energy that has been thermalized is smaller for the large convertor than the smaller convertor. As an example, at $6 \mathrm{~ns}$ approximately $1 / 2$ of the kinetic energy of the $z$-pinch has been thermalized for the small convertor, but only about $1 / 4$ of the kinetic energy has been thermalized for the large convertor. This is because the shock velocity is about the same in both cases but the convertor density is approximately 2.4 times higher for the small convertor.

\section{B. Copper Mass Scan}

An inertial fusion capsule could be imbedded in a solid foam convertor as was studied in the last sub-section. However, the initial low temperature $\left(\mathrm{T}_{\mathrm{rad}} \sim 100 \mathrm{eV}\right)$ radiation wave needed to set the capsule implosion on a proper adiabat can arrive at the capsule equator significantly before it arrives at the poles. This is because the radiation wave travels at much less than the speed of light even in a low density plastic foam. This timing difference would result in an asymmetric implosion, without some design mitigation such as a quasi-spherical pinch. The arrival asynchrony could be substantially reduced by using hollow convertors, since the radiation wave would then move at the speed of light. Such a hollow convertor must have an initial radius large enough so that the capsule implodes before the convertor collapses on the capsule. Initial model calculations and detailed numerical simulations suggest that the hohlraum temperature should be a weak function of the ratio of the convertor mass over the $z$-pinch plasma mass and that increasing this ratio should delay stagnation on axis. This would allow a smaller initial radius of the convertor and thus a higher hohlraum temperature.

A series of dynamic hohlraum experiments were performed on the $\mathrm{Z}$ accelerator to test this hypothesis. Four shots were performed using the nested wire array configuration described in the last sub-section. The convertor consisted of a $1 \mathrm{~cm}$ long copper tube with a $2.5 \mathrm{~mm}$ radius. 
The wall thickness was different for each shot to provide a scan in the ratio of the convertor to $z$-pinch mass. The wall thicknesses were $1.8,3.7,9.2$, and $9.4 \mu \mathrm{m}$, with the corresponding ratios $\mathrm{M}_{C} / \mathrm{M}_{\mathrm{Z}}=0.8,1.6,4.0$, and 4.1. The hohlraum temperature was determined by measuring $\mathrm{x}$-rays through a $2 \mathrm{~mm}$ radius aperture on one end of the dynamic hohlraums. The results are shown in Fig. 11 as plots of hohlraum temperature as a function of $M_{C} / M_{Z}$. These data (symbols) are compared to calculated temperatures using our model (solid line). The agreement is pretty good for small mass ratios; however, the data exhibits a somewhat stronger dependence on the mass ratio than predicted by the model. This may be due to the opacity of copper, which has not been included in the model. An optimum convertor should be of a low opacity material such as plastic foam.

\section{Model results compared to detailed numerical simulations}

The model equations described above are easy to solve numerically. An important benefit of such a computationally simple model is that it is easy to obtain many solutions and thus uncover various trends. In the previous section we obtained the hohlraum temperature as a function of the ratio of the convertor/z-pinch mass, $M_{C} / M_{Z}$ for copper convertors, see Fig. 11 . Notice that the hohlraum temperature is a weak function of the mass ratio. This is because increasing the convertor mass extracts more energy from the inelastic collision (see eq. (19)), but more energy goes into internal energy.

We shall now look at the variations with respect to the initial radius of the z-pinch plasma. The convertor is still assumed to be a thin ( $2 \mathrm{~mm}$ thick) annulus, but composed of a plastic foam which has less opacity than copper. For each value of the initial radius of the z-pinch plasma, the mass ratio $\mathrm{M}_{C} / \mathrm{M}_{Z}$ is optimized to obtain the highest hohlraum temperature. The resulting hohlraum temperature (normalized to $\mathrm{T}_{\mathrm{opt}}=134 \mathrm{eV}$ ) is plotted in Fig. 12 as a function of the initial radius of the $\mathrm{z}$-pinch, $\mathrm{r}_{0}$. In this example the capsule radius is $1 \mathrm{~mm}$, the drive current rises to $20 \mathrm{MA}$ in $100 \mathrm{~ns}$, the z-pinch plasma is $2 \mathrm{~mm}$ thick just prior to first strike, and $F_{\mathrm{s}}=r_{\mathrm{zib}} / \mathrm{r}_{\text {cap }}=2$. The initial convertor radius determined by eq. (10) is found to be approximately $5 \mathrm{~mm}$. Notice that the hohlraum temperature is a fairly strong function of the initial z- 
pinch plasma radius for values below optimum. This is because small initial radii require large z-pinch plasma masses to maintain the correct implosion time and thus most of the kinetic energy goes into internal energy rather than radiation. The function is weak for initial radii greater than optimum because the reduction in mass is compensated by the increase in the radiation lost, since the degree of radiation trapping is reduced. However, this weak behavior probably does not continue for arbitrarily large initial wire radius, due to the RT instability. One would expect that a RT bubble would break through the z-pinch plasma if the initial radius is too large. Furthermore, the optical depth of the z-pinch plasma is less than unity for the large initial radii so the diffusion approximation is no longer valid. Fortunately, this is clearly not the region of interest.

In our next example, the symmetry factor, $\mathrm{F}_{\mathrm{s}}$, is varied, while the initial radius and the mass ratio $M_{C} / M_{Z}$ are both optimized. The results are shown in Fig. 13. Two curves are plotted. Parameters corresponding to the performance that can be expected from the existing Z-accelerator were used for the dotted curve. Here the current rises to a peak value of $20 \mathrm{MA}$ in $100 \mathrm{~ns}$ and the capsule radius is $1 \mathrm{~mm}$. The model indicates that $\mathrm{r}_{0}=2 \mathrm{~cm}, \mathrm{M}_{\mathrm{C}} / \mathrm{M}_{\mathrm{Z}}=2$, and $\mathrm{M}_{\mathrm{Z}} / \mathrm{L}_{\mathrm{Z}}=$ $3 \mathrm{mg} / \mathrm{cm}$ are near optimum. Parameters corresponding to the performance that could be expected from an advanced accelerator were used to generate the solid curve. Here the current rises to $60 \mathrm{MA}$ in $100 \mathrm{~ns}$ and the capsule radius is assumed to be $2 \mathrm{~mm}$. Optimum parameters are found to be; $\mathrm{r}_{\mathrm{z} 0}=3 \mathrm{~cm}, \mathrm{M}_{\mathrm{C}} / \mathrm{M}_{Z}=3, \mathrm{M}_{Z} / \mathrm{L}_{\mathrm{Z}}=15 \mathrm{mg} / \mathrm{cm}$. We have omitted the correction for the RT instability, $F_{R T}$, so that these results could be directly compared to 1-D numerical simulations using the detailed radiation hydrodynamics code LASNEX ${ }^{9}$. The optimized values of the initial radius, z-pinch mass and the convertor mass were used to set up these simulations. The convertor was chosen to be $\mathrm{CH}$ plastic foam, with an initial inner radius set to $r_{z i b}$, and an initial outer radius determined by eq. (11). A thin layer of gold $(0.4 \mu \mathrm{m})$ on the outside of the convertor was found to reduce the ablation in response to the roughly $30 \mathrm{eV}$ radiation generated in the z-pinch plasma during the implosion phase. The z-pinch plasma (tungsten) was initiated with a thickness of $1 \mathrm{~mm}$ as an approximation to the conditions expected after the wire array undergoes the initial ohmic heating. An external circuit was used to generate the z-pinch current with lumped circuit elements $(\mathrm{R} \sim 1 / 8 \mathrm{ohm}, \mathrm{L}=12 \mathrm{nH})$ and a time-dependent voltage. 
The current rose to a peak.value of $20 \mathrm{MA}$ for $\mathrm{Z}$ parameters (dotted curve) and $60 \mathrm{MA}$ for a hypothetical next generation $\mathrm{X}$ machine (solid curve) in approximately $100 \mathrm{~ns}$. The implosions occurred at about $135 \mathrm{~ns}$. Absorption of radiation by the capsule was simulated by a radiation leak of the same surface area as each respective capsule. The average hohlraum temperatures over the capsule implosion time are plotted as diamonds for $\mathrm{Z}$ parameters and as squares for $\mathrm{X}$ parameters. As can be sen there is good agreement between these simulations and the model calculations. This illustrates the usefulness of such a computationally simple model, since performing the double optimization with a detailed simulation code would take a long time. The results of Fig. 13 indicate that decreasing the value of $F_{s}$ substantially increases the hohlraum temperatures. However, the simulations show that the convertor will be driven into the capsule before it is fully imploded if $F_{s}$ is too small. The numerical simulations indicate that $F_{s}$ must be greater than 1.5 to hydrodynamically isolate the capsule. This would limit the peak hohlraum temperature to approximately $230 \mathrm{eV}$, when the effect of the RT instability is included, even with a $60 \mathrm{MA}$ drive current. Smaller values of $\mathrm{F}_{\mathrm{s}}$ and hence higher temperatures are possible using solid foam convertors because of a phenomenon we call ablative standoff. This is described in the next section.

\section{Scaling Results}

\section{A. Ablative Standoff}

A low density solid foam convertor has two important advantages over thin annular convertors. First, the fraction of the collision energy that goes into shock heating the z-pinch plasma decreases as the convertor-density is decreased, see eq. (23). This will decrease the outward radiation losses and result in higher hohlraum temperatures. Second, the transit time of the shock through the convertor can be as long as the implosion time of the capsule. This continuous heating can result in a rising hohlraum temperature during the capsule implosion. Proper density profiling of the convertor should result in "pulse shaping" which can drive an isen-

tropic implosion. In fact, numerical simulations ${ }^{5}$ indicate that a nearly isentropic implosion results without any density profiling. Furthermore, these simulations indicate that the z-pinch 
plasma/convertor does not crush the capsule even though the radius of the interface is less than the original capsule radius at the time of the capsule implosion, i.e. $F_{\mathrm{s}}<1$. This is in spite of the fact that near the electrodes a shock wave has been driven all the way to the axis before the capsule implosion. The shock wave does not destroy the symmetry of the capsule due to a phenomenon that we shall call "ablative standoff". The shock is isolated from the imploding portion of the capsule by the outward moving material that has been ablated from the capsule by the hohlraum radiation field. This shock isolation or "ablative standoff" only occurs if the ablation ram pressure is larger than the shock pressure. The equations describing this process are given in section II $\mathrm{C}$. The general requirements are high radiation temperatures and low convertor densities. Since the capsule symmetry is not adversely affected by the incoming shock, ablative standoff allows small values of $F_{s}$, which implies higher hohlraum radiation temperatures. This is true for several reasons. More z-pinch kinetic energy is available when the pinch is driven to a smaller final radius. Less radiation is lost because of the smaller final radius. Furthermore, the incoming shock will be reflected from both the axis and the capsule ablation material. This outward reflected shock will further slow down the incoming convertor and $z$-pinch plasma turning this kinetic energy into heat. Since the shock reflection occurs earlier at the capsule equator than elsewhere, the z-pinch plasma will wrap around the capsule forming a somewhat spherical hohlraum. These effects all conspire to produce a hotter hohlraum than would be produced by an annular convertor.

The results of a model calculation assuming a peak drive current of $55 \mathrm{MA}$, a risetime of $100 \mathrm{~ns}$, and an initial z-pinch radius of $1.8 \mathrm{~cm}$ is shown in Fig. 14. These parameters are the same as those used in the recent high yield design ${ }^{5}$. The results are plotted as a function of the ratio $\mathrm{M}_{\mathrm{C}} / \mathrm{M}_{\mathrm{Z}}$. The peak hohlraum temperature has a maximum of approximately $260 \mathrm{eV}$ for $\mathrm{M}_{\mathrm{C}} / \mathrm{M}_{\mathrm{Z}} \sim 0.8$. This is substantially below the value of about $300 \mathrm{eV}$ that was observed in detailed numerical simulations of the high yield capsule ${ }^{5}$. However, the numerical simulations did not include the effect of the Rayleigh-Taylor instability and radiation loss to the electrode surfaces. Indeed, removing these effects from our model increases the calculated peak temperature to approximately $290 \mathrm{eV}$, which is close to the results of the simulations. Improvements have been made to the simulation code that will allow the inclusion of these effects and one of 
us (J.S.L.) will revisit these design calculations. It will be interesting to see if the peak temperature is reduced by a significant amount as our model predicts.

The ratio of ablation standoff radius to the capsule radius, $r_{a b} / r_{c a p}$, is a decreasing function of $\mathrm{M}_{\mathrm{C}} / \mathrm{M}_{Z}$, because the initial shock pressure depends on the convertor density. The number of shock transit times, $\mathrm{N}_{\mathrm{S}}$, is approximately three, which means that a second shock will usually reach the capsule before it is fully imploded. This shock will be stronger than the first shock due to the higher convertor density and could possibly damage the-symmetry of the capsule. However, the hohlraum temperature will be roughly at its peak value when the second shock arrives and the ablation pressure may still be able to standoff this second shock. We do not think it is practical to include the standoff of the second shock in our simple model. However, the ablation pressure was sufficient to provide standoff of the second shock in the detailed numerical simulations ${ }^{5}$. The energy accounting is shown in Fig $14 \mathrm{c}$ ). The kinetic energy of the $z$-pinch was approximately $7 \mathrm{MJ}$ and is not a function of the ratio $\mathrm{M}_{C} / \mathrm{M}_{Z}$. However, the energy available for heating monotonically rises with $M_{C} / M_{Z}$. This is the reason for the initial rise in the hohlraum temperature as $\mathrm{M}_{\mathrm{C}} / \mathrm{M}_{\mathrm{Z}}$ increases. The temperature reaches a maximum and then starts to fall slowly because of the extra mass that must be heated. This can be seen from the behavior of the curve labeled, $E_{\mathbb{I N T}}$.

The behavior of the dynamic hohlraum as the initial radius of the z-pinch is varied is shown in Fig. 15, where, $I_{p}=55 \mathrm{MA}, r_{\text {cap }}=2.75 \mathrm{~mm}$, and $F_{s}=0.6$ The optimum convertor mass is found for each value of the initial radius, $r_{\mathrm{z} 0}$. For small initial radii, an increase in the initial radii increases the hohlraum temperature because the energetics is dominated by the internal energy, $E_{\mathbb{N N T}}$, which decreases due to the smaller z-pinch mass. Continuing to increase the initial radii results in too small a $z$-pinch mass to effectively trap the radiation. This effect is seen in the functional behavior of the outer temperature of the z-pinch plasma at capsule bang time, $T_{o b}$, the optical depth, and the radiation energy lost from the outer surface of the z-pinch plasma, $E_{S}$. The maximum hohlraum temperature occurs for an initial radius of approximately $1.9 \mathrm{~cm}$, which happens to be very close to the value used for the high yield design. 
The hohiraum temperature as a function of the drive current for optimized values of the convertor mass and the initial z-pinch radius is shown in Fig. 16. A capsule the size of the high yield capsule, i.e. $r_{\text {cap }}=2.75 \mathrm{~mm}$ has been assumed. The optimum ratio $\mathrm{M}_{C} / \mathrm{M}_{Z}$ is found to be nearly constant at 0.8 so we have not plotted it. The available energy rises as the square of the drive current, but due to the fourth power dependence of the radiation loss the hohlraum temperature rises approximately as the square root of the current. The degree of radiation trapping increases with drive current as seen by the number of optical depths, $\tau$, in the $z$-pinch plasma. The fraction of the available energy that is lost to radiation and that is absorbed by the capsule is nearly independent of the drive current. The optimal initial radius of the convertor only increases from $1.2 \mathrm{~cm}$ at $I_{p}=20 \mathrm{MA}$ to $1.5 \mathrm{~cm}$ at $I_{p}=60 \mathrm{MA}$, but this results in a significant change in the foam density. A foam density of $2 \mathrm{mg} / \mathrm{cc}$ is optimal at $20 \mathrm{MA}$, which is the current that can be produced by the existing $Z$ accelerator. However, the minimum foam density the can be can be produced by the present state of the art is approximately $5 \mathrm{mg} / \mathrm{cc}$, so the optimum design may not be practical at low current drives. Notice also that the ablation standoff radius is nearly the capsule radius at the lower current drives. Reducing the capsule radius to $1.5 \mathrm{~mm}$ results in higher radiation temperatures and optimal foam densities $\left(\mathrm{r}_{\mathrm{co}} \sim 0.7 \mathrm{~cm}\right)$ as seen in Fig. 17, but the ablative standoff is still marginal. Clearly detailed numerical simulations and experiments will be needed to determine if ablative standoff can be used with current drives less than about $40 \mathrm{MA}$.

\section{B. Radiation Trapping}

A significant uncertainty affecting the performance of the dynamic hohlraum is the degree of radiation trapping that will be achieved by the z-pinch plasma once it implodes onto the convertor. The computation of the opacity of high $\mathrm{Z}$ materials is extremely difficult and thus prone to error. Furthermore, as discussed in section II E, the RT instability will reduce the effective optical depth of the z-pinch plasma due to the bubble and spike structure. In this section, we explore the sensitivity of the dynamic hohlraum performance to such uncertainties in the degree of radiation trapping. For this study, we have multiplied the opacity in our model calculations by the variable factor $F_{O P}$ rather than the fixed factor $F_{R T} \sim 0.37$. The results are plotted 
in Fig. 18. The solid lines are for advanced accelerator performance as would be expected on the proposed $\mathrm{X}$ machine, i.e. a drive current of $55 \mathrm{MA}$ and a capsule radius of $2.75 \mathrm{~mm}$. The dotted curves are performance that could be expected on the existing $\mathrm{Z}$ machine, i.e. a drive current of $20 \mathrm{MA}$ and a capsule radius of $1.5 \mathrm{~mm}$. The results indicate that the hohlraum temperature is reduced by approximately $10-15 \mathrm{eV}$ when $\mathrm{F}_{\mathrm{OP}}=\mathrm{F}_{\mathrm{RT}} \sim 0.37$, which corresponds to the RT corrections that have been made in the previous section. As might be expected the performance falls off faster as $F_{O P}$ decreases. As seen in Fig. 18b, the fraction of the available energy for heating that is lost by radiation increases as $F_{O P}$ decreases. This fraction is larger for the $Z$ parameters because the mass of the z-pinch is much smaller. The ratio $r_{a b} / r_{c a p}$ indicates strength of the ablative standoff, which is considerably larger for $\mathrm{X}$ parameters. We assume that ablative standoff will not be effective when $\mathrm{r}_{\mathrm{ab}} / \mathrm{r}_{\text {cap }}<1$. It should be noted that stagnation of the z-pinch plasma against the convertor may increase $F_{O P}$ above our estimated value of 0.37 with a significant benefit to the hohlraum performance. It may also be worth using opacity cocktails to improve radiation trapping.

\section{Axial Convergence}

Axial convergence of an initially cylindrical z-pinch will occur if the z-pinch material has an appropriate axial mass profile, i.e. heavier at the midplane lighter at the ends. The determination of the initial mass profile that will provide a quasi-spherical final configuration can most readily be found by trial and error. Since this could require many iterations, we developed a simple 2-D numerical model of an imploding z-pinch. We assume azimuthal symmetry and represent the $z$-pinch as a number of particles (actually rings). We assume a fixed current wave form typical of a z-pinch shot on the existing $\mathrm{Z}$ accelerator and calculate the $\mathbf{I} \mathbf{x} \mathbf{B}$ force on each of these particles and then move them accordingly. The model ignores the effects of material pressure. Furthermore, the evolution of the pinch can only be simulated until a pair of particles crosses. Thus a relatively small number (10-15) of particles can be used. Despite these limitations, the model has proved to be very useful for finding mass distributions that result in quasi-spherical final configurations. The results of such a simulation are shown in Fig. 3. The position of particles, marked with the symbol + , are shown at various times during the implo- 
sion. Initially pinch was cylindrical with a radius of $2 \mathrm{~cm}$ and axial length of $1 \mathrm{~cm}$. The initial mass/length, $\Lambda$, is given by eq. (1). As can be seen the final shape is nearly spherical, which should provide better coupling to the spherical inertial fusion capsule. Similar results have been obtained by others ${ }^{7}$. There are several potential advantages to using an axially convergent or "quasi-spherical" z-pinch to drive a dynamic hohlraum. First, the outer surface area of a quasi-spherical z-pinch is smaller than for a cylindrical pinch, which reduces radiation losses. Second, the areal density of a quasi-spherical z-pinch is larger than a cylindrical z-pinch of the same mass. This further reduces radiation losses. Third, the nearly spherical final configuration should provide better macroscopic radiation uniformity (not referring to the RT effects discussed earlier).

Detailed numerical simulations will be needed to determine if an axial convergent $z$-pinch can improve the radiation uniformity, but we have used our model to predict the improvement in hohlraum temperature that results from the first two advantages. The results are shown in Fig. 19. The results indicate an increase of approximately $20 \mathrm{eV}$ nearly independent of the drive current. Although this may not seem like a large improvement, one must remember that the radiation intensity is proportional to the fourth power of the temperature, so this represents an improvement in the radiation intensity of $35 \%$ at $60 \mathrm{MA}$ and $75 \%$ at $20 \mathrm{MA}$. Inspection of Fig. $19 b$ reveals that the foam density is substantially lower for the quasi-spherical z-pinches. This is because the initial convertor radii are about the same in either the cylindrical or the quasi-spherical pinches, but there is greater volume compression in the quasi-spherical pinches. Presently the lowest foam densities that can be fabricated are approximately $5 \mathrm{mg} / \mathrm{cc}$. To determine the importance of this limitation on the fabrication of foam convertors, we have calculated the hohlraum temperature with the convertor density fixed at $5 \mathrm{mg} / \mathrm{cc}$. The results are shown in Fig. 20. We have chosen a capsule radius of $1.25 \mathrm{~mm}$ for these calculations because the model predicts that larger capsules will not exhibit ablative standoff at drive currents as low as $20 \mathrm{MA}$ with this foam density. The results indicate that the temperature increase in a quasi-spherical pinch is approximately the same even if the foam is higher than optimal, i.e. $5 \mathrm{mg} / \mathrm{cc}$. Of course, the temperatures for both cylindrical and quasi-spherical are somewhat lower. 
There is another potential problem with the axially convergent z-pinches. The initial mass profile used to shape the pinch as it implodes, also seeds the Rayleigh-Taylor instability. Some numerical details of the seeding and the growth of the instability are worked out in Appendix III. To investigate the stability behavior of mass profiled $z$-pinches we have performed 2-D numerical simulations with the MHD code $\mathrm{MACH} 2^{17}$. This code calculates all three components of the velocity and magnetic field vectors on a Arbitrary Lagrangian/Eulerian (ALE) mesh. The electron, ion, and radiation temperatures are calculated. Generalized Ohm's law and tabular equations of state are used. An important feature of the code is that it can handle complex shapes. Density contours at a time near final collapse for two simulations are shown in

Fig. 21. The initial mass/length profiles were calculated using eq. (1). The coefficient $\mathrm{a}_{2}=-0.06$ for both simulations. The coefficient $a_{4}=0.024$ for the upper half of the density contour plot, while $a_{4}=0.026$ for the lower half. Note the clear development of the Rayleigh-Taylor instability even though no random density seed was used in these calculations. In contrast, simulations performed with uniform mass/length and no random seed show no development of instability. Furthermore, the two simulations show significant variation even though the mass/length varied by only $0.2 \%$ between these two simulations. These simulations suggest that the z-pinch mass profile must be achieved with considerable accuracy to obtain a desired configuration. These simulations used a mesh size of $156 \mu$. The effect of the RT instability is considerably larger for simulations performed with half this cell size as seen in Fig. 22. These results clearly demonstrate that obtaining a well behaved z-pinch implosion with axial convergence by tailoring the mass profile may be very difficult. Since axially convergent pinches should increase the peak hohlraum temperatures and may help improve radiation symmetry the approach should be tested experimentally. However, considerable care will be needed to insure the mass profile is created with sufficient accuracy.

\section{Conclusions}

We have presented a quasi-analytic model of the dynamic hohlraum, which includes most of the important physics for determining the hohlraum radiation temperature. The model includes the z-pinch implosion dynamics, the conversion of the z-pinch energy into heat and 
radiation, the loss of radiation to the electrode walls and through the z-pinch plasma, and radiation absorbed by the capsule. This model has been used to determine the expected performance scaling (peak hohlraum temperature) of the dynamic hohlraum as a function of the many parameters that determine this system. As an example, the model has been used to compute hohlraum temperatures within dynamic hohlraum experiments using a solid foam convertor. The results are in good agreement with the experimental data. Calculations of the peak hohlraum temperature as a function the ratio of the convertor mass to the $\mathrm{z}$-pinch mass have also been compared to experimental data. The agreement with this data, which was obtained using annular copper convertors, is not quite as good as for the foam convertors, but still within about $10 \mathrm{eV}$.

The model has been used to find the optimum values of the ratio of the convertor mass to the z-pinch mass and the initial z-pinch radius for annular convertors. Detailed 1-D Lasnex simulations using these optimized values compared well to the results of our model. The results show that the peak hohlraum temperature is a strong function of the ratio of the radius of the interface between the z-pinch plasma and the convertor over the capsule radius. Smaller values result in higher temperatures. We have presented Lasnex and view factor calculations that indicate adequate radiation symmetry can be obtained if this ratio remains larger than two, which corresponds to $F_{s}=r_{z i b} / r_{\text {cap }}>0.5$. Note that since the capsule is imploding while the zpinch plasma is moving inward, the minimum radius of the interface, $r_{z i b}$, can be smaller than the initial radius of the capsule, $\mathrm{r}_{\text {cap }}$. Small values of $\mathrm{F}_{\mathrm{s}}$ leads to high peak hohlraum temperatures, which are suitable for driving fusion capsules. If radiation temperature variations induced by the RT instability are larger $(>30 \%)$ or the wavelengths longer $(\sim 1 \mathrm{~mm})$ than indicated by the Lasnex simulations, the symmetry factor will need to be larger, with an associated decrease in the peak hohlraum temperature. Therefore, the determination of the wavelength and amplitude of the radiation source variations produced within the convertor by the RT instability is a critical importance toward determining if the dynamic hohlraum concept is a viable approach to inertial confinement fusion. Experiments are planned to determine both the wavelength and the radiation temperature variations induced by the RT instability. 
We have used the model to investigate dynamic hohlraum performance using a solid foam convertor, which has been used in a recent high yield design ${ }^{5}$. The Lasnex calculations of the high yield design show a phenomenon that we call "ablative standoff". The inward shock wave generated by the z-pinch plasma colliding with the foam convertor does not penetrate to the inside of the capsule because of the outward motion of the radiatively ablated material. We have calculated the radius of the standoff point as a function of drive current and capsule size. We find that ablative standoff works well at the high current drives needed for a high yield capsule, but not as well at the lower current drives that are presently available. Thus it may be difficult to demonstrate this phenomenon without an upgrade to the existing accelerator.

Scaling of the hohlraum temperature with the degree of radiation trapping has been determined using the model. The results indicate that a decrease in the effective optical depth of the $z$-pinch plasma by a factor of two results in a decrease of about $10 \mathrm{eV}$ in the peak hohlraum temperature. The degree of trapping might be improved by using a cocktail of materials to increase the opacity. This would be most beneficial for demonstrating ablative standoff with relatively low current drives.

A z-pinch will undergo axial convergence if the initial z-pinch has an appropriate mass profile. The final shape near stagnation can be nearly spherical (quasi-spherical). We have used the model to estimate the performance advantage of quasi-spherical z-pinches over purely cylindrical pinches. The results indicate that the quasi-spherical dynamic hohlraum should reach a temperature of roughly $20 \mathrm{eV}$ higher than a purely cylindrical pinch. This result is nearly independent of the drive current. Unfortunately, we also show that the mass profile needed to produce axial convergence provides a seed for the Rayleigh-Taylor instability. We demonstrate this both analytically and numerically. Experiments should be performed to determine if this approach can be made to work.

Acknowledgments: We wish to thank Thomas Mehlhorn for helpful discussions and encouragement during the course of this work. We also acknowledge the technical help of the Z-crew and Paul Mix for help with the graphics. This work performed at Sandia National Laboratories. Sandia is a multiprogram laboratory operated by Sandia Corporation, a Lockheed 
Martin Company, for the United States Department of Energy under Contract No. DE-AC0494AL85000

\section{Appendix I: Analytic z-pinch solution}

Analytic solutions for cylindrical and axially convergent $z$-pinches are derived in this appendix. The equation of motion for a cylindrical z-pinch is given by the expression

$$
\mathrm{M}_{\mathrm{Z}} \frac{\mathrm{d}^{2} \mathrm{r}_{\mathrm{z}}}{\mathrm{dt}^{2}}=-\frac{\mathrm{B}^{2}}{2 \mu_{0}} \mathrm{~A}_{\mathrm{z}}\left(\mathrm{r}_{\mathrm{z}}\right),
$$

where $\mathrm{M}_{\mathrm{Z}}$ is the total mass of the $\mathrm{z}$-pinch plasma, $\mathrm{r}_{\mathrm{Z}}$ is the radius of the pinch, $\mathrm{B}$ is the strength of the B-field produced by the z-current, $B=\frac{\mu_{0} I}{2 \pi r}$. To include z-pinch implosions with axial convergence we use the expression for the area

$$
A_{z}\left(r_{z}\right)=2 \pi r_{z} L_{z}\left(\frac{r_{z}}{r_{z 0}}\right)^{\alpha}
$$

where $L_{Z}$ is the length of the pinch, $\alpha=0$ corresponds to cylindrical convergence, and $\alpha=1 / 3$ corresponds to the axial convergent pinch depicted in Fig. 3. A solution to Eq. A1.1 is found by using the trial solution

$$
r_{z}=r_{z 0}\left(1-\left(\frac{t}{t_{p}}\right)^{\beta}\right)^{\zeta}
$$

where $t_{p}$ is the time to stagnation. Substitution into eq. A1.1, reveals that the choice $\beta=4$, and $\zeta=1$ yields a solution with a drive current that rises linearly with time as is typically observed in the experiments. The result is

$$
\begin{gathered}
I^{2}=I_{p}^{2} h(\alpha)\left(\frac{t}{t_{p}}\right)^{2}\left(1-\left(\frac{t}{t_{p}}\right)^{4}\right)^{1-\alpha}, \\
r_{z}=r_{z 0}\left(1-\left(\frac{t}{t_{p}}\right)^{4}\right)
\end{gathered}
$$


where $I_{p}$ is the peak current, and $h(\alpha)=\frac{(3-2 \alpha)^{\frac{3}{2}-\alpha}}{(2-2 \alpha)^{1-\alpha}}$

The normalized drive current and $z$-pinch radius for a cylindrical pinch $(\alpha=0)$ are shown in Fig. 4. The rise time to peak current is $t_{r}=\frac{t_{p}}{(3-2 \alpha)^{1 / 4}}$, the pinch mass/length is

$$
\frac{M_{Z}}{L_{Z}}=f(\alpha)\left(\frac{\mu_{0}}{4 \pi}\right)\left(\frac{I_{p} t_{r}}{r_{z 0}}\right)^{2}
$$

where $f(\alpha)=\frac{(3-2 \alpha)^{2-\alpha}}{12(2-2 \alpha)^{1-\alpha}}$. The kinetic energy of the pinch is

$$
E_{K}=. g(\alpha) L_{Z}\left(\frac{\mu_{0}}{4 \pi}\right) I_{p}^{2}\left(1-\frac{r}{r_{z 0}}\right)^{3 / 2}
$$

where $g(\alpha)=\frac{2(3-2 \alpha)^{\frac{3}{2}-\alpha}}{3(2-2 \alpha)^{1-\alpha}}$. The velocity of the pinch can be found easily from eq. A1.5. The result is

$$
v_{z}\left(r_{z}\right)=\frac{4 r_{z 0}}{t_{p}}\left(1-\frac{r_{z}}{r_{z 0}}\right)^{3 / 4}
$$

We have compared the analytic solution for the kinetic energy of the pinch to a numerical solution with lumped circuit elements which approximates the current provided by the $\mathrm{Z}$ accelerator. The voltage is assumed to be of the form $\mathrm{V}=5.5 \sin \left(\frac{\pi \mathrm{t}}{2 \mathrm{t}_{\mathrm{r}}}\right) \mathrm{MV}$, which drives current through a series inductance of $11.4 \mathrm{nH}$ and resistance of $0.12 \mathrm{ohms}$. The results of this comparison is shown in Fig. 5 The agreement is very good up to a convergence ratio of approximately 10. Since the dynamic hohlraum stagnates on a convertor the convergence ratio $(\sim 4)$ is substantially less than 10 and the analytic solution should not introduce significant error.

\section{Appendix II: radiation transport solution}

The z-pinch plasma is of high opacity so that it can effectively trap the radiation. Therefore the diffusion approximation is appropriate with the radiation flux given by the expression 


$$
F=-\frac{4 \sigma}{3 \kappa \rho} \frac{d}{d r} T_{R}^{4}
$$

where $\sigma$ is the Stefan-Boltzmann constant, $k$ is the opacity, $T_{R}$ is the radiation temperature, and $\rho$ is the density. In steady state energy conservation yields the expression

$$
\nabla \cdot F=\varepsilon
$$

The specific power, $\varepsilon$, deposited in the z-pinch plasma is given by the expression

$$
\varepsilon=\frac{f_{Z} E_{S}}{V_{Z} \tau_{r s}}
$$

where $V_{Z}$ is the volume of the z-pinch plasma, $E_{A}$ is given by eq. (19) or eq. (21) and $E_{\mathbb{N N T}}$ is given by eq. (25). For cylindrical geometry $V_{Z}=\pi\left(r_{z o b}^{2}-r_{z i b}^{2}\right) L_{Z}$ and for spherical geometry $\mathrm{V}_{\mathrm{Z}}=\frac{4}{3} \pi\left(\mathrm{r}_{\mathrm{zob}}^{3}-\mathrm{r}_{\mathrm{zib}}^{3}\right)$. We shall assume that $\varepsilon$ is constant and thus Eq. A2.2 can be integrated.

The radiation flux within the z-pinch plasma for cylindrical geometry is

$$
F=\frac{\varepsilon \mathrm{r}}{2}+\frac{1}{r}\left(\frac{E_{S}}{2 \pi L_{Z} \tau_{r s}}-\frac{\varepsilon r_{\text {zob }}^{2}}{2}\right)
$$

where the condition $F\left(r_{z o b}\right) 2 \pi r_{z o b} L_{Z}=\frac{E_{S}}{t_{r s}}$ has been used to determine the constant of integration.

The radiation flux for spherical geometry is

$$
\mathrm{F}=\frac{\varepsilon \mathrm{r}}{3}+\frac{1}{\mathrm{r}^{2}}\left(\frac{\mathrm{E}_{\mathrm{S}}}{4 \pi \tau_{\mathrm{rS}}}-\frac{\varepsilon \mathrm{r}_{\mathrm{zob}}^{3}}{3}\right)
$$

where the condition $F\left(r_{z o b}\right) 4 \pi r_{z o b}^{2}=\frac{E_{S}}{t_{r s}}$ has been used to determine the constant of integration.

$\operatorname{Lindl}^{8}$ gives the power law fit to the opacity of gold 


$$
\kappa=\kappa_{0} \mathrm{~T}_{\mathrm{hev}}^{-\beta} \rho^{0.3},
$$

where $\beta=1.5, \kappa_{0}=6 \times 10^{3} \mathrm{~cm}^{2} / \mathrm{g}$, and $\mathrm{T}_{\text {hev }}$ is the temperature in units of $100 \mathrm{eV}$. This should be a reasonably good approximation for the opacity of tungsten. Using this relation eq. A2.1 can be put in the form

$$
F=-\chi \frac{d}{d r} T_{R}^{4+\beta},
$$

where $\chi=\frac{16 \sigma}{3 \bar{\kappa}(4+\beta)}$ and $\bar{\kappa}=\kappa_{0} \rho^{1.3}$. Eqs. A2.4 and A2.7 can be combined and integrated. The result for cylindrical geometry is

$$
\chi \mathrm{T}_{\mathrm{R}}^{4+\beta}=\frac{\varepsilon\left(\mathrm{r}_{\mathrm{zob}}^{2}-\mathrm{r}^{2}\right)}{4}+\left(\frac{\mathrm{E}_{\mathrm{S}}}{2 \pi \mathrm{L}_{\mathrm{Z}} \tau_{\mathrm{rs}}}-\frac{\varepsilon \mathrm{r}_{\mathrm{zob}}^{2}}{2}\right)\left[\ln \left(\frac{\mathrm{r}_{\mathrm{zob}}}{\mathrm{r}}\right)\right]+\chi \mathrm{T}_{\mathrm{o}}^{4+\beta},
$$

where $T_{0}$ is the radiation temperature at the outer surface of the $z$-pinch plasma. The result for spherical geometry is

$$
\chi \mathrm{T}_{\mathrm{R}}^{4}+\beta=\frac{\varepsilon\left(\mathrm{r}_{\mathrm{zob}}^{2}-\mathrm{r}^{2}\right)}{6}+\left(\frac{\mathrm{E}_{\mathrm{S}}}{4 \pi \tau_{\mathrm{rs}}}-\frac{\varepsilon \mathrm{r}_{\mathrm{zob}}^{3}}{3}\right)\left(\frac{1}{\mathrm{r}}-\frac{1}{\mathrm{r}_{\mathrm{zob}}}\right)+\chi \mathrm{T}_{\mathrm{o}}^{4+\beta}
$$

\section{Appendix III: Axial Convergence and the Rayleigh-Taylor Instability}

In this appendix we show that a nonuniform mass profile can seed the Rayleigh-Taylor (RT) instability. The mass profile can be expanded in a Fourier series

$$
\Lambda(z)=\Lambda_{0}\left(1+a_{2} z^{2}\right)=\sum_{n=0}^{\infty}\left(A_{n} \cos (2 \pi n z)+B_{n} \sin (2 \pi n z)\right),
$$

where we have only retained the dominant quadratic term in eq. (1). The Fourier coefficients are $A_{0}=\Lambda_{0}\left(1+\frac{a_{2}}{3}\right), A_{n}=\frac{\Lambda_{0} a_{2}}{(n \pi)^{2}}$, and $B_{n}=\frac{\Lambda_{0} a_{2}}{n \pi}$.

The one dimensional equation of motion for a cylindrical pinch is

$$
\frac{\mathrm{d}^{2} \mathrm{r}_{\mathrm{z}}}{\mathrm{dt}^{2}}=-\frac{\mu_{0} \mathrm{I}^{2}}{4 \pi \mathrm{r}_{\mathrm{z}} \Lambda}
$$


Solutions to this equation for constant mass/length, $\Lambda$, are found in the Appendix I. The result is $r_{z}(\tau)=r_{z 0}\left(1-\tau^{4}\right)$ where $\tau=\frac{t}{t_{p}}$. We can find solutions for small variations in $\Lambda$ by linearization. Let $r_{z}(z, \tau)=r_{z}(\tau)+\Delta(\tau) e^{i k z}$ and $\Lambda(z)=\Lambda_{0}\left(1+\tilde{a} e^{i k z}\right)$, where $k=2 \pi / \lambda$. The first order equation is then

$$
\tilde{\delta}\left(1-\tau^{4}\right)-12 \delta=12 \tau^{2}\left(1-\tau^{4}\right),
$$

where the derivative is with respect to $\tau$ and $\delta=\Delta / r_{z 0}$. The equation has the solution

$$
\delta=\tilde{a}\left(\tau^{4}-\frac{2}{5} \tau^{6}+O\left(\tau^{8}\right)\right)
$$

The spatial modes will grow exponentially when they reach sufficient amplitude. Using the 1$D$ cylindrical solution for the acceleration, a, and the Takabe formula ${ }^{8} \gamma=\sqrt{\frac{k a}{1+k L_{s}}}$ for the $\mathrm{RT}$ growth rate we find

$$
\int \gamma \mathrm{dt}=\sqrt{\frac{12 \pi n r_{z 0}}{\mathrm{~L}_{\mathrm{z}}+4 \pi \mathrm{nL}_{s}}} \tau^{2}
$$

where $L_{s}$ is the effective density scale length of the $z$-pinch plasma. Comparing this to the algebraic solution we find that exponential growth takes over at time

$$
\tau_{e}=\left(\frac{2\left(L_{Z}+4 \pi n L_{s}\right)}{6 \pi n r_{z 0}}\right)^{1 / 4}
$$

Since $L_{s}$ is small compared to $r_{z 0}$, and $L_{Z}$ is small compared to $3 r_{z 0}$, exponential growth occurs at times significantly smaller than the collapse time. 


\section{Appendix IV: symbol definitions}

$a=$ acceleration

$a_{2}$ and $a_{4}=$ second and fourth order coefficients for mass/length of a $z$-pinch

$\alpha=$ axial convergence parameter ( $\alpha=0$ for cylindrical pinches)

$\alpha_{c}$ and $\alpha_{w}=$ capsule and electrode wall albedos

$A_{z}=$ area of the $z$-pinch

$A_{n}$ and $B_{n}=$ Fourier coefficients to expand the mass/length of a z-pinch

$\beta_{s}=$ average shock velocity over the average velocity of $z$-pinch plasma

$\beta=$ coefficient for power law fit to the opacity of gold

$\mathrm{d}_{\mathrm{z}}$ and $\mathrm{d}_{\mathrm{c}}=$ thickness of the $\mathrm{z}$-pinch plasma and the convertor at initial contact

$\varepsilon=$ specific power within the z-pinch plasma

$\mathrm{E}_{\mathrm{A}}=$ energy available for heating after the collision

$\mathrm{E}_{\text {cap }}=$ the energy absorbed by the capsule .

$E_{\text {int }}=$ energy that goes into internal degrees of freedom

$\mathrm{E}_{\mathrm{K}}=$ kinetic energy of $\mathrm{z}$-pinch plasma just before collision

$E_{\text {rad }}=$ energy that is radiated from the dynamic hohlraum before capsule bang time

$\mathrm{E}_{\mathrm{s}}=$ energy radiated from the outer surface of the $\mathrm{z}$-pinch plasma

$E_{w}=$ radiation energy absorbed by the electrode wall

$E_{z}(T)$ and $E_{c}(T)=$ internal energy functions for z-pinch plasma and the convertor

$F_{\text {op }}=$ opacity multiplier

$\mathrm{F}_{\mathrm{s}}=$ symmetry factor $=\mathrm{r}_{\mathrm{zib}} / \mathrm{r}_{\text {cap }}$

$\mathrm{F}_{0}=$ radiation source flux for view factor calculations without Rayleigh-Taylor

$\delta \mathrm{F}=$ radiation source flux for view factor calculations with Rayleigh-Taylor

$\delta F_{1}=$ radiation source flux that produces $1 \%$ flux variation on the capsule

$\mathrm{f}_{\mathrm{z}}=$ fraction of the shock heating that goes into the $\mathrm{z}$-pinch plasma

$\gamma=$ Gruneisen equation of state coefficient

$\Gamma(\mathrm{z})=$ thickness of the $\mathrm{z}$-pinch plasma with variations due to Rayleigh-Taylor

$\Gamma_{\min }=$ minimum thickness of the z-pinch plasma

$\Gamma_{\text {Ross }}=$ Rosseland mean of the z-pinch plasma over thickness variations due to RT

$\mathrm{I}_{\mathrm{p}}=$ peak drive current

$\kappa=$ opacity

$\Lambda(\mathrm{z})=$ mass/length of a $\mathrm{z}$-pinch

$\Lambda_{0}=$ zeroth order term in a Taylor expansion of the mass/length of a z-pinch

$\mathrm{L}_{\text {hohl }}=$ length of hohlraum for view factor radiation symmetry calculations

$\mathrm{L}_{\mathrm{s}}=$ density scale length for $\mathrm{RT}$ growth rate

$\lambda=$ wave length of the Rayleigh-Taylor instability

$M_{\text {cap }}=$ initial capsule mass

$\mathrm{m}_{\text {cap }}(\mathrm{r})=$ unablated capsule mass as a function of radius as it implodes

$\mathrm{M}_{\mathrm{c}}=$ mass of the convertor

$\mathrm{M}_{\mathrm{z}}=$ mass of the $\mathrm{z}$-pinch plasma

$\mathrm{N}_{\mathrm{s}}=$ number of times a shock is driven through the convertor before capsule bang time

$\mu=$ shock convergence coefficient

$\mathrm{P}_{\text {ram }}=$ ram pressure generated by ablated material interacting with the convertor

$\sigma=$ Stefan-Boltzmann constant

$r_{a b}=$ ablation stand off radius 
$r_{\text {cap }}=$ initial capsule radius

$r_{c}(t)=$ capsule radius as a function of time

$\mathrm{R}_{\mathrm{ZH}}=$ ratio of the average $\mathrm{z}$-pinch plasma temperature over the hohlraum temperature

$\mathrm{R}_{\text {hohl }}=$ hohlraum radius for view factor radiation symmetry calculations

$\mathrm{r}_{\mathrm{z} 0}=$ initial radius of the $\mathrm{z}$-pinch

$r_{z}(t)=$ radius of the $z$-pinch as a function of time

$r_{\mathrm{zi}}(t)$ and $r_{\mathrm{zo}}(t)=$ radius of the inner/outer surface of the $z$-pinch as a function of time

$r_{z i b}$ and $r_{z o b}=$ inner/outer radius of $z$-pinch plasma at capsule bang time

$r_{c o}$ and $r_{c i}=$ initial outer/inner radius of the convertor

$\rho_{\mathrm{c}}=$ initial convertor mass density

$\mathrm{T}_{\mathrm{o}}(\mathrm{t})=$ outer temperature of the $\mathrm{z}$-pinch plasma as a function of time

$\mathrm{T}_{\mathrm{ob}}=$ outer temperature of the $\mathrm{z}$-pinch plasma at capsule bang time

$\tau_{b}=$ capsule bang time

$\tau_{\text {cap }}=$ capsule implosion time

$t_{p}=$ time to pinch to the axis

$t_{\mathrm{T}}=$ rise time to peak current

$\tau_{\mathrm{r}}=$ rise time to peak peak hohlraum temperature for annular convertors

$\tau_{\mathrm{rc}}=$ effective radiation absorption time for the capsule

$\tau_{\mathrm{rw}}=$ effective radiation absorption time for the electrode wall

$\tau_{\mathrm{rs}}=$ effective radiation emission time for loss from the outer surface of the z-pinch plasma

$\mathrm{T}_{\mathrm{HP}}=$ peak hohlraum radiation temperature

$\mathrm{T}_{\mathrm{H}}(\mathrm{t})=$ hohlraum radiation temperature as a function of time

$\mathrm{T}_{\mathrm{R}}(\mathrm{r})=$ radiation temperature within the $\mathrm{z}$-pinch plasma

$v_{s} \bar{v}_{s}=$ shock velocity and average shock velocity

$v_{z}$ and $\bar{v}_{z}=z$-pinch velocity and average $z$-pinch velocity

$\mathrm{V}_{\mathrm{z}}=$ volume of the $\mathrm{z}$-pinch plasma 


\section{References}

1 R. B. Spielman et. al. Phys. of Plasmas, 5, 2105, 1998.

2 J. L. Porter, Bull. Am. Phys. Soc. 42, 1948 (1998): R. J. Leeper et. al. Nuclear Fusion, 39, $1283,1999$.

3 J. H. Hammer, M. Tabak, S. C. Wilks, J. D. Lindl, D. S. Bailey, P. W. Rambo, A. Toor, G. B. Zimmerman, Phys. Plasmas, 6, 2129, 1999.

4 J. H. Brownell, R. L. Bowers, Bull. Am Phys. Soc. 40, 1848 (1995)

5 J. Lash et. al. "The Prospects for High Yield ICF with a Z-Pinch Driven Dynamic Hohlraum", Proceedings of IFSA, Bordeaux, FR, Sept. 1999

6 J. H. Brownell, R. L. Bowers, K. D. Mclenithan, and D. L. Peterson, Phys. Plasmas, 5, 2071,1998

7 I. V. Lisitsyn, S. Katsuki, and H. Akiyama, Phys. Plasmas, 1389, 1999.

8 John Lindl. Phys. Plasmas, 2, 3933, 1995.

9 G. B. Zimmerman and W. L. Kruer, "Numerical simulation of laser initiated fusion," Comments Plasma Phys. 2, 51 (1975).

10 Y. B. Zel'dovich and Y. P. Raizer "Physics of Shock Waves and High-Temperature Hydrodynamic Phenomenon" Academic Press, New York, 1966

11 G. B. Whitham, "Linear and Nonlinear Waves", John Wiley and Sons, New York, p. 198, 1974.

12 K. S. Holian, "T-1 Handbook of Material Properties Data Bases", Los Alamos National Laboratory, LA-10160-MS UC-34 Nov. 1984

13 M. P. Desjarlais and B. M. Marder, Phys. Plasmas, 6, 2057,1999.

14 R.J. Leeper et. al. " $Z$ pinch driven inertial confinement fusion target physics research at Sandia National Laboratories" 17th IAEA Fusion Energy Conference, Yokohama, Japan, 
October, 1998, Nuclear Fusion, 39, 1283, Yokohama Special Issue 1, 1999.

15 C. Deeney, M. R. Douglas, R. B. Spielman, T.J. Nash, D.L. Peterson, P.L'Eplattenier, G.A. Chandler, J.F. Seaman, and K.W. Struve, Phys. Rev. Lett. 81, 4883, 1998

16. T. J. Nash et al., Rev. Sci. Instrum. 70, 464, 1999.

17 R.E. Peterkin Jr., M.H. Frese, and C.R. Sovinec, J. Comp. Phys. 140, 148, 1998. 


\section{Figures}

1 A schematic of a double-ended z-pinch-driven hohlraum

2 A schematic of a dynamic hohlraum. The z-pinch plasma is shown at the time of first impact with the convertor. The initial inner and outer radius of the convertor are labeled $r_{c i}$ and $r_{c o}$.

3 Numerical solution for axially convergent $z$-pinch at several times. The simplified model is described in the text.

$4 \quad$ Normalized drive current and $z$-pinch radius for a cylindrical pinch

5 A comparison between the analytic solution (dotted) and a numerical solution (solid) for the kinetic energy of a cylindrical z-pinch

6 Results of a Lasnex simulation of a z-pinch stagnating onto a plastic convertor, a) radiation temperature contours, b) line out of the density as a function of axial distance $z$.

7 Source variation that yields a $1 \%$ variation at the capsule as calculated by view factor simulations.

8 Schematic of z-pinch plasma deformed by the RT instability just before striking the convertor.

9 Hohlraum radiation as a function of time for shot 291 (symbols) compared to model calculations (solid line). The foam convertor had an initial radius of $4 \mathrm{~mm}$ with a density of $6 \mathrm{mg} / \mathrm{cc}$. 
10 Hohlraum radiation as a function of time for shot 297 (symbols) compared to model calculations (solid line). The foam convertor had an initial radius of $2.5 \mathrm{~mm}$ with a density of $14.3 \mathrm{mg} / \mathrm{cc}$.

11 Hohlraum temperature as a function of the ratio $\mathrm{M}_{C} / \mathrm{M}_{Z}$ for annular copper convertors. The results of the model calculations are labeled $T_{H P}$ for the peak hohlraum temperature and $\mathrm{T}_{\mathrm{o}}$ for the temperature at the outside of the $\mathrm{z}$-pinch plasma. The diamonds are experimental measurements of the hohlraum temperature.

12 Normalized hohlraum temperatures $\left(\mathrm{T}_{\mathrm{H}} / \mathrm{T}_{\mathrm{OPT}}\right)$ as a function of the initial z-pinch plasma radius for annular $2 \mathrm{~mm}$ thick plastic foam convertors. The mass ratio of the $\mathrm{M}_{C} / \mathrm{M}_{Z}$ is optimized for each value of $\mathrm{r}_{z 0}$. The available energy $\left(E_{A}\right)$, radiated energy $\left(E_{\mathrm{rad}}\right)$, and internal energy, $\left(E_{\mathrm{int}}\right)$ are also plotted

13 Hohlraum temperature as a function of $F_{s}=r_{z i b} / r_{\text {cap }}$ for annular $2 \mathrm{~mm}$ thick foam convertors. The dotted curve corresponds to performance of the existing Z-accelerator $\left(I_{p}=20 \mathrm{MA}, r_{\text {cap }}=1 \mathrm{~mm}\right)$. The diamonds are the result of Lasnex simulations for the optimized parameters obtained from the model. The solid curve corresponds to an advanced accelerator $\left(I_{p}=60 \mathrm{MA}, r_{\text {cap }}=2 \mathrm{~mm}\right)$. The squares are the results of Lasnex simulations for these optimized parameters.

14 Results from model calculations assuming a solid foam convertor with a peak drive current of $55 \mathrm{MA}$, a z-pinch mass of $48 \mathrm{mg}$, an initial z-pinch radius of 1.8 $\mathrm{cm}$, a z-pinch kinetic energy of $7 \mathrm{MJ}$, a capsule radius of $2.75 \mathrm{~mm}$, and a symmetry factor $F_{s}=0.6$. All parameters are plotted as a function of $M_{c} / M_{z}$. The peak hohlraum temperature, $T_{H P}$ and outer surface temperature of the convertor, $T_{0}$, are plotted in a). The ratio of the ablation standoff radius, $r_{a b}$, to the initial capsule radius, $r_{c a p}$, and the number of shocks passing through the convertor, $N_{S}$, is plotted in b). The ratios of the energy available for heating, $E_{A}$, the energy lost from the outer surface of the pinch as radiation, $E_{s}$, the energy absorbed by the capsule, 
$E_{\text {cap }}$, and the internal energy, $E_{i n t}$, over the kinetic energy of the incoming z-pinch plasma, $E_{K}$, are plotted in $\left.c\right)$.

15 Results from model calculations assuming a solid foam convertor with a peak drive current of $55 \mathrm{MA}$, a capsule radius of $2.75 \mathrm{~mm}$, and a symmetry factor $\mathrm{F}_{\mathrm{sym}}=0.6$. Parameters for optimal convertor mass are plotted as a function of the initial zpinch radius, $r_{\mathrm{zO}}$. The peak hohlraum temperature, $\mathrm{T}_{\mathrm{HB}}$ and outer surface temperature of the convertor, $\mathrm{T}_{0}$, are plotted in a). The optical depth with the Rayleigh-Taylor factor included is plotted in $b$ ). The energy available for heating, $E_{A}$, the energy lost from the outer surface of the pinch as radiation, $E_{s}$, the energy absorbed by the capsule, $E_{\text {cap }}$, and the internal energy, $E_{\text {int }}$ are plotted in $\left.c\right)$.

16 Optimized results (both convertor mass and initial z-pinch radius) from model calculations assuming a solid foam convertor are plotted as a function of the peak drive current, $I_{p}$ assuming a capsule of $2.75 \mathrm{~mm}$, and a symmetry factor $F_{s}=0.6$. The peak hohlraum temperature, $\mathrm{T}_{\mathrm{HP}}$ and outer surface temperature of the convertor, $T_{0}$, are plotted in a). The optical depth with the Rayleigh-Taylor factor included and the optimum foam density are plotted in b). The energy available for heating, $E_{A}$, the energy lost from the outer surface of the pinch as radiation, $E_{S}$, and the energy absorbed by the capsule, $E_{\text {cap }}$ are plotted in $\left.c\right)$. The optimal initial $z$-pinch radius, $r_{\mathrm{z} 0}$ and the ratio of the ablation standoff radius to the capsule radius, $\mathrm{r}_{\mathrm{ab}} / \mathrm{r}_{\text {cap }}$ is plotted in $\mathrm{d}$ ).

17 Same parameters as Fig. 16 but assuming a capsule radius of $1.5 \mathrm{~mm}$.

18 Optimized results as a function of the opacity multiplier $\mathrm{F}_{\mathrm{op}}$, see text for description. The solid curves labeled $\mathrm{X}$ are results assuming a peak drive current of 55 MA and a capsule radius of $2.75 \mathrm{~mm}$. The dotted curves labeled $\mathrm{Z}$ are results assuming a peak drive current of $20 \mathrm{MA}$ and a capsule radius of $1.5 \mathrm{~mm}$. The hohlraum temperature is plotted in a), the fraction of the available energy that goes into 
radiation is plotted in b), and ratio of the ablative standoff radius to the initial capsule radius is plotted in c).

19 Comparison between cylindrical (labelled CYL) and quasi-spherical (labelled QS) dynamic hohlraum model calculations. A capsule radius of $2.75 \mathrm{~mm}$ is assumed and $\alpha=1 / 3$ for the quasi-spherical calculation. Hohlraum temperatures are plotted in a) and convertor densities in b).

20 Comparison between cylindrical and quasi-spherical dynamic hohlraum temperature model calculations assuming a fixed convertor density of $5 \mathrm{mg} / \mathrm{cc}$. A capsule radius of $1.25 \mathrm{~mm}$ is assumed and $\alpha=1 / 3$ for the quasi-spherical calculation.

21 Contour plots of the z-pinch plasma density near final collapse as calculated in two separate simulations using the 2-D MHD code, $\mathrm{MACH}$, with slightly different initial mass profiles. The initial density profiles were determined by eq. (1) with $a_{2}=-0.06$ and $a_{4}=0.024$ for the simulation displayed in the upper half and $a_{4}=0.026$ for the simulation displayed in the lower half. Mirror symmetry about $\mathrm{z}=0$ has been assumed for both simulations.

22 Contour plots of the z-pinch plasma density from two different MACH2 simulations using different cell resolutions. The results using $156 \mu$ cells are displayed in the upper half, while the results using $78 \mu$ cells is displayed in the lower half. The initial density profile was the same for both simulations as determined by eq. (1) with $\mathrm{a}_{2}=-0.06$ and $\mathrm{a}_{4}=0.024$. 


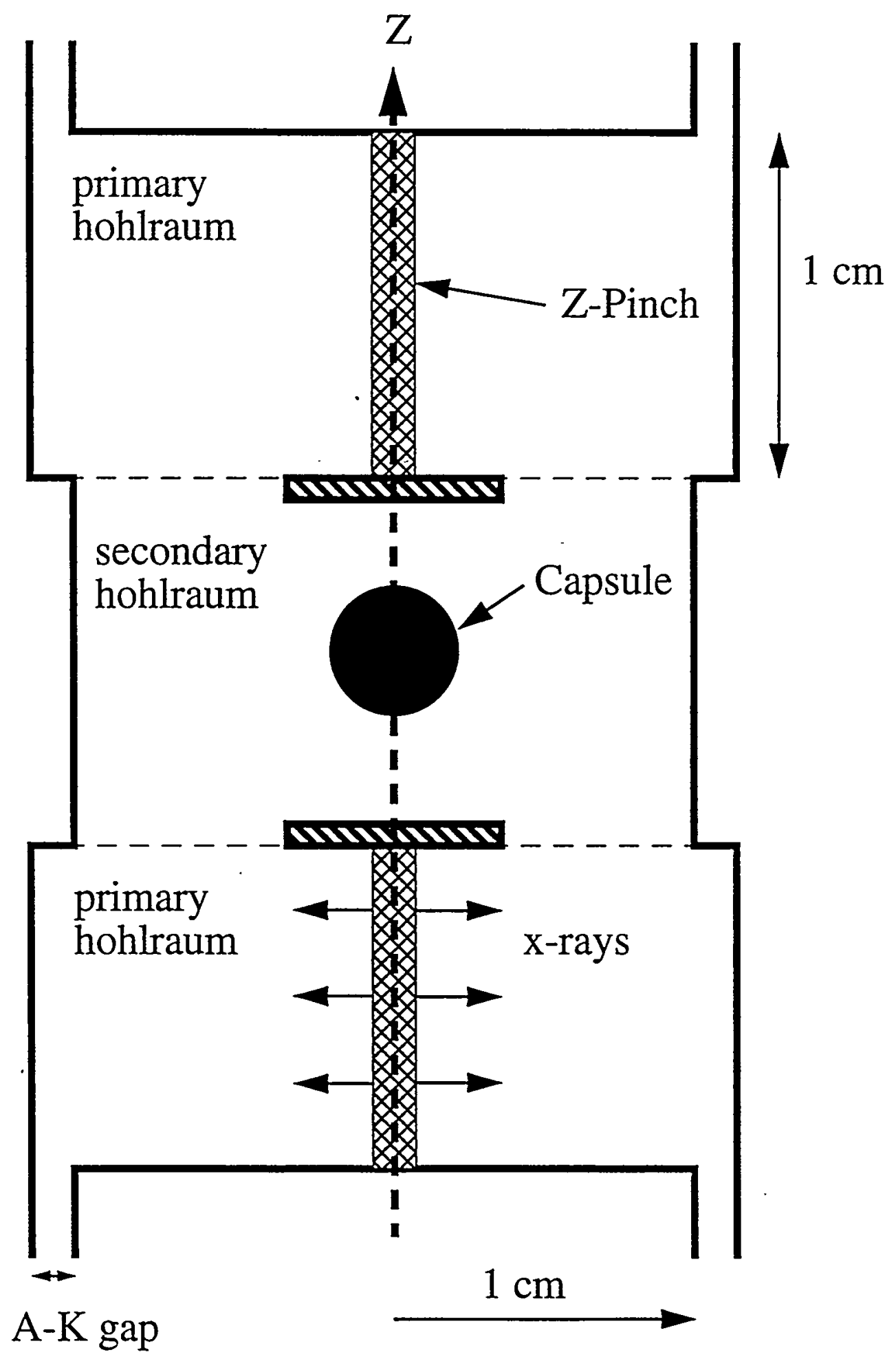

Fig 1 


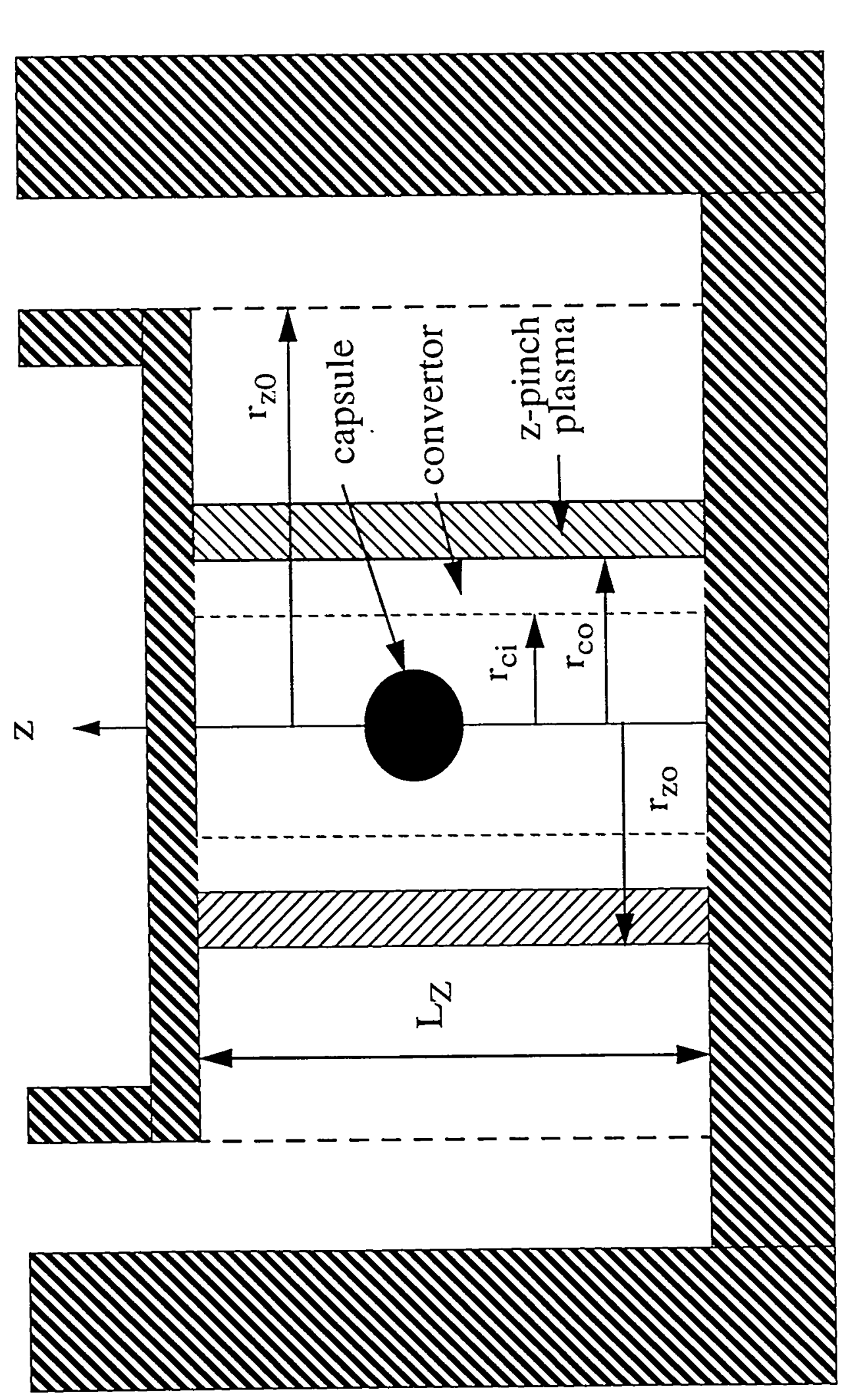

N

OD 


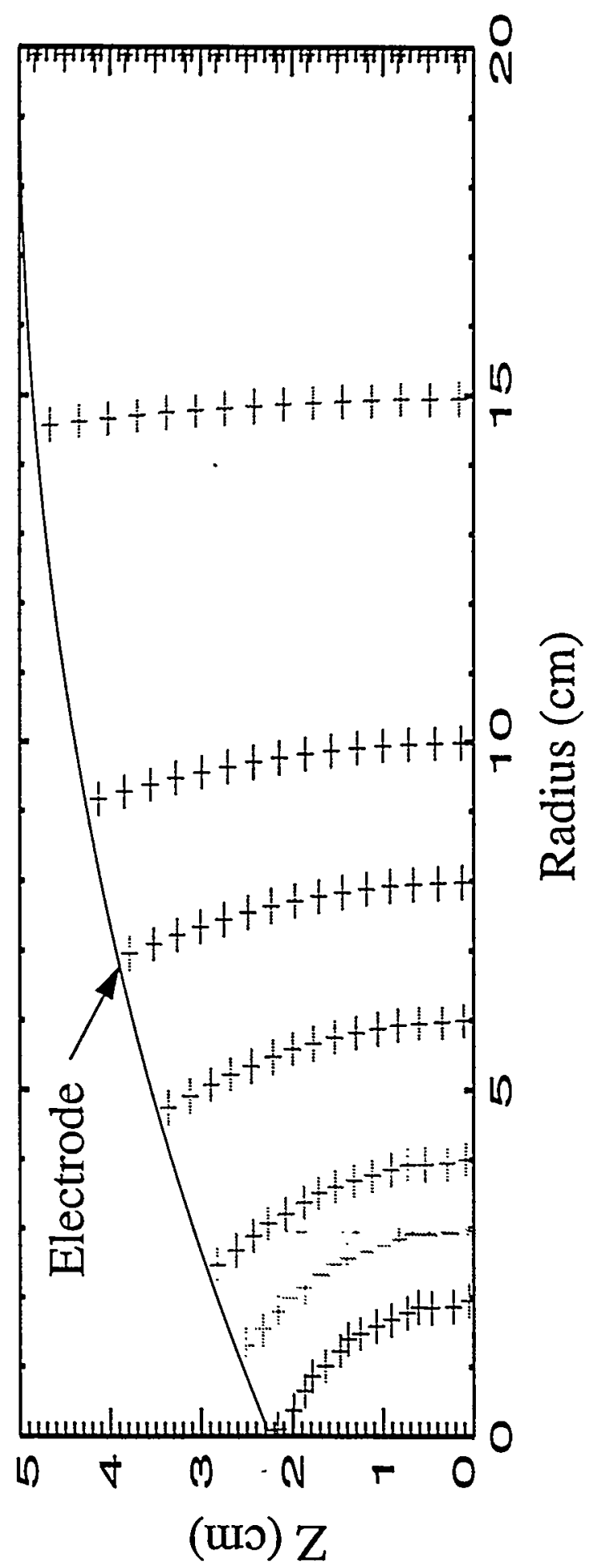

$m$

$\dot{0}$ 


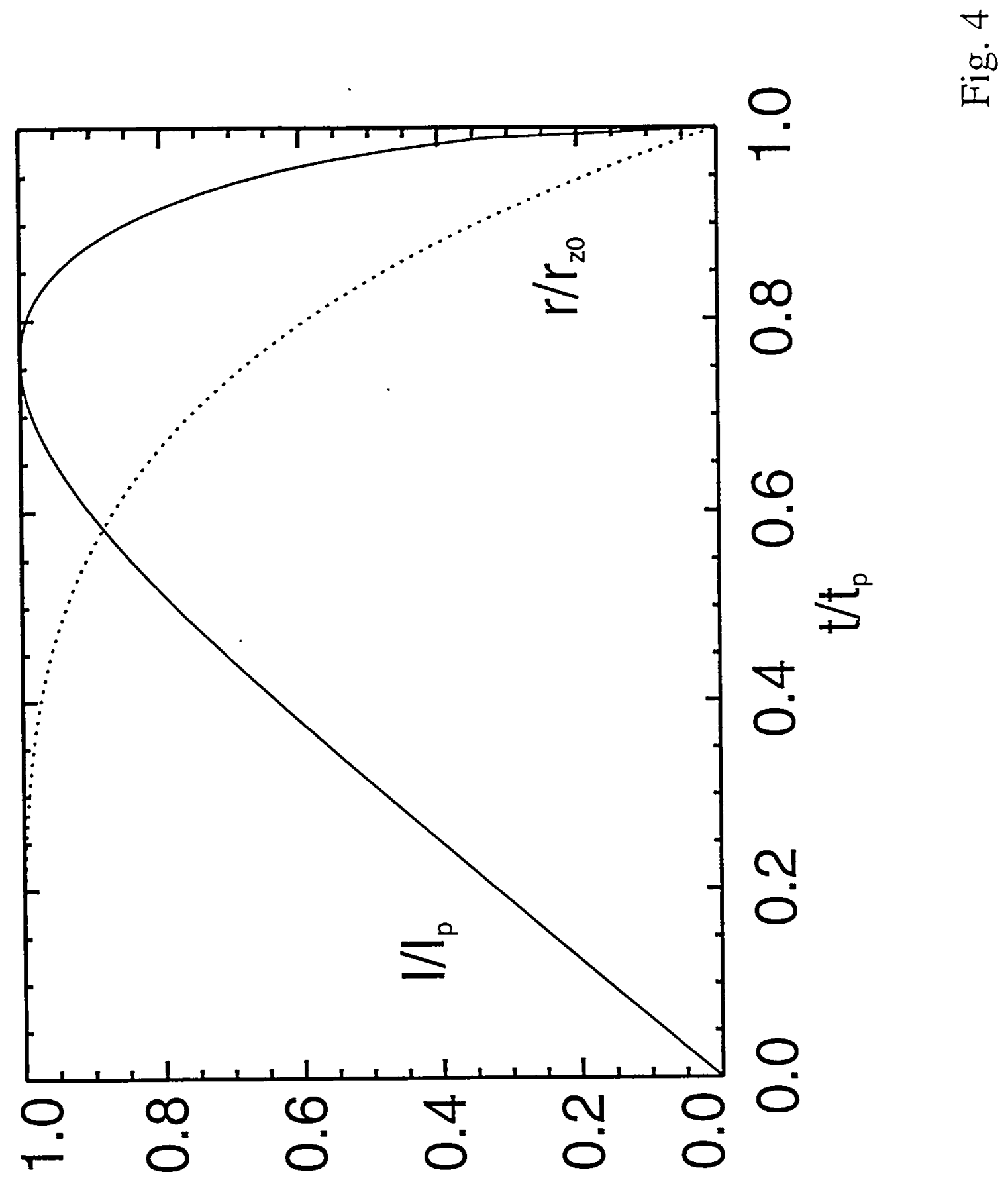


$n$
$\dot{0}$

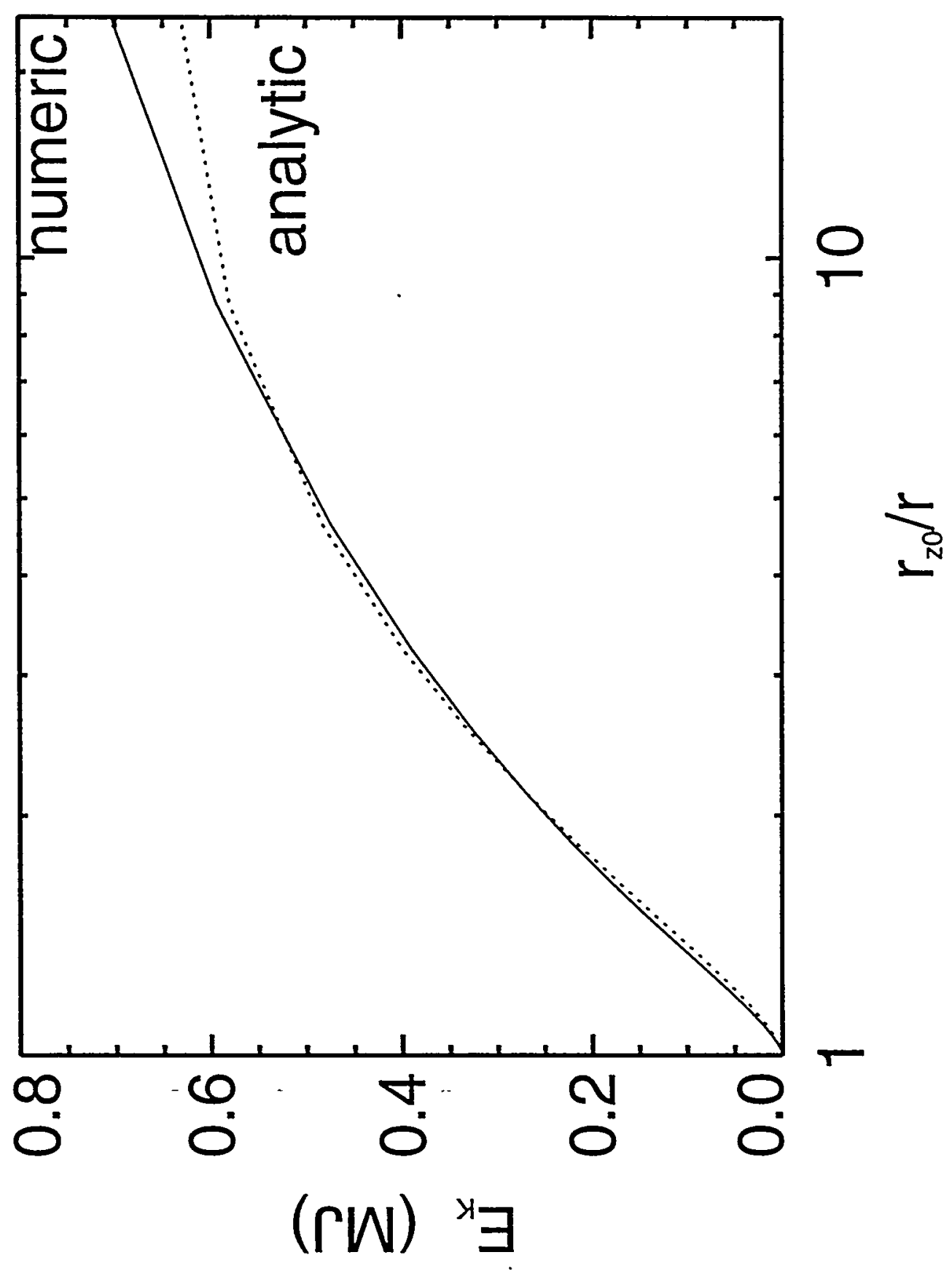




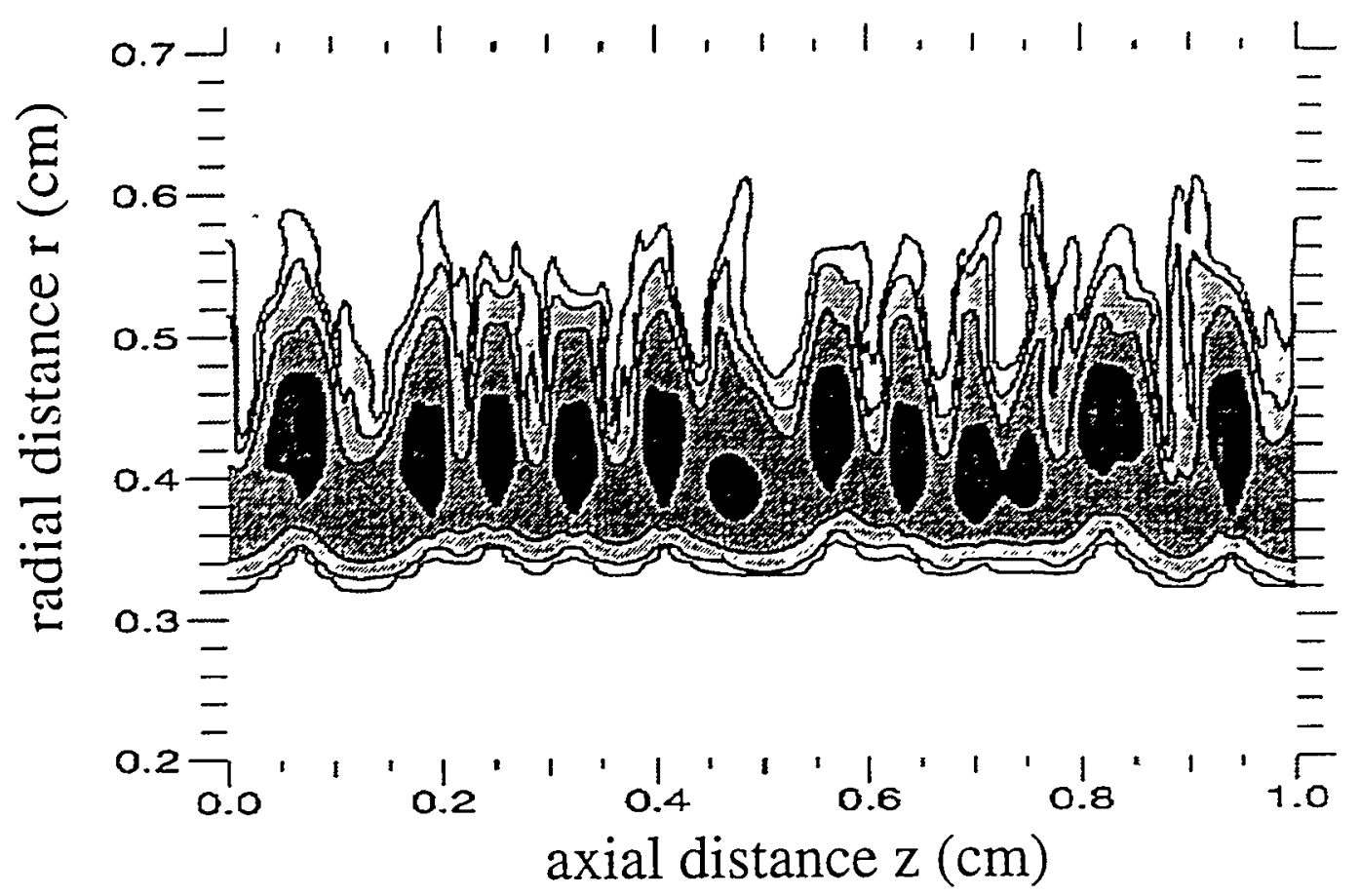

a)
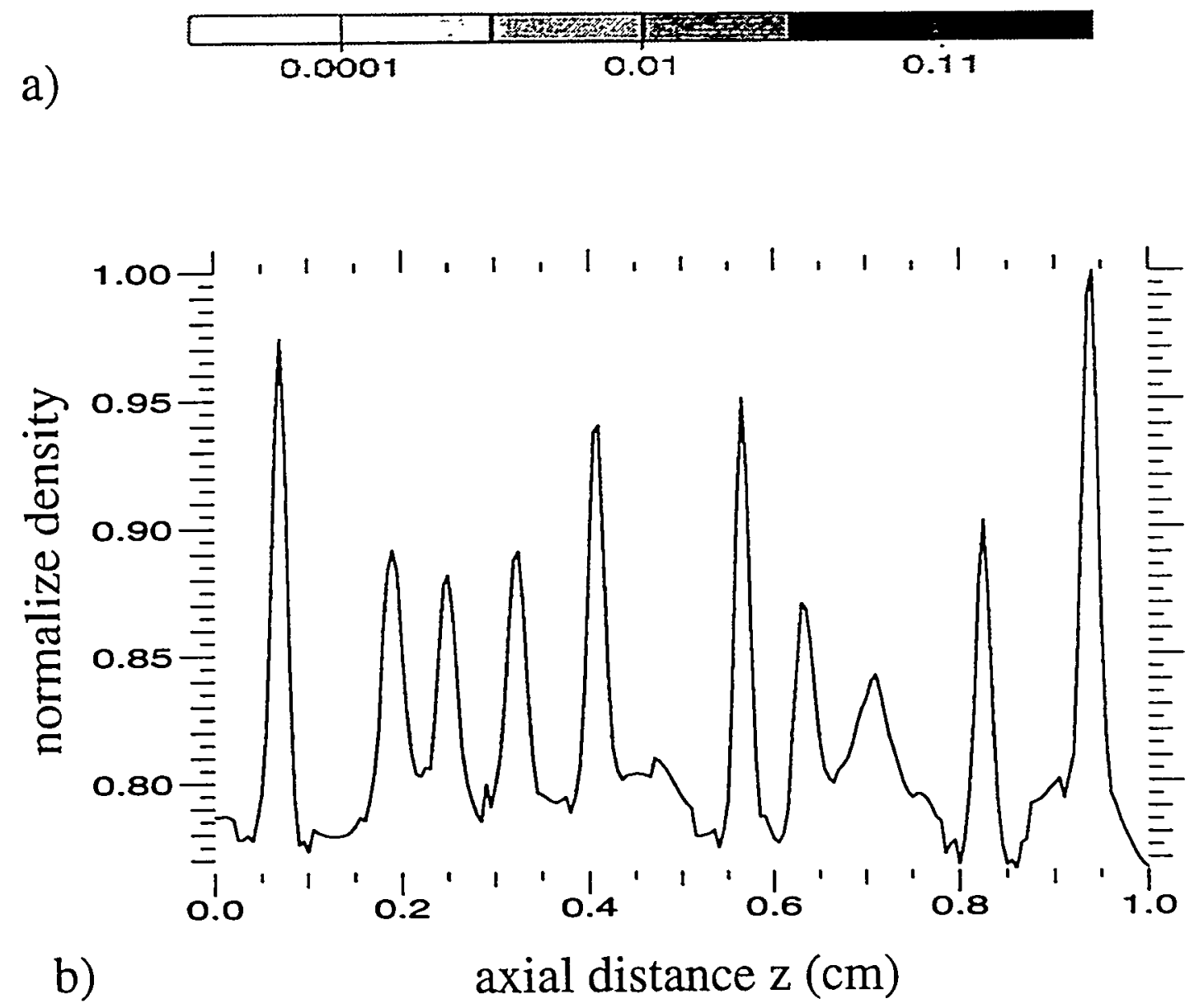

Fig 6 


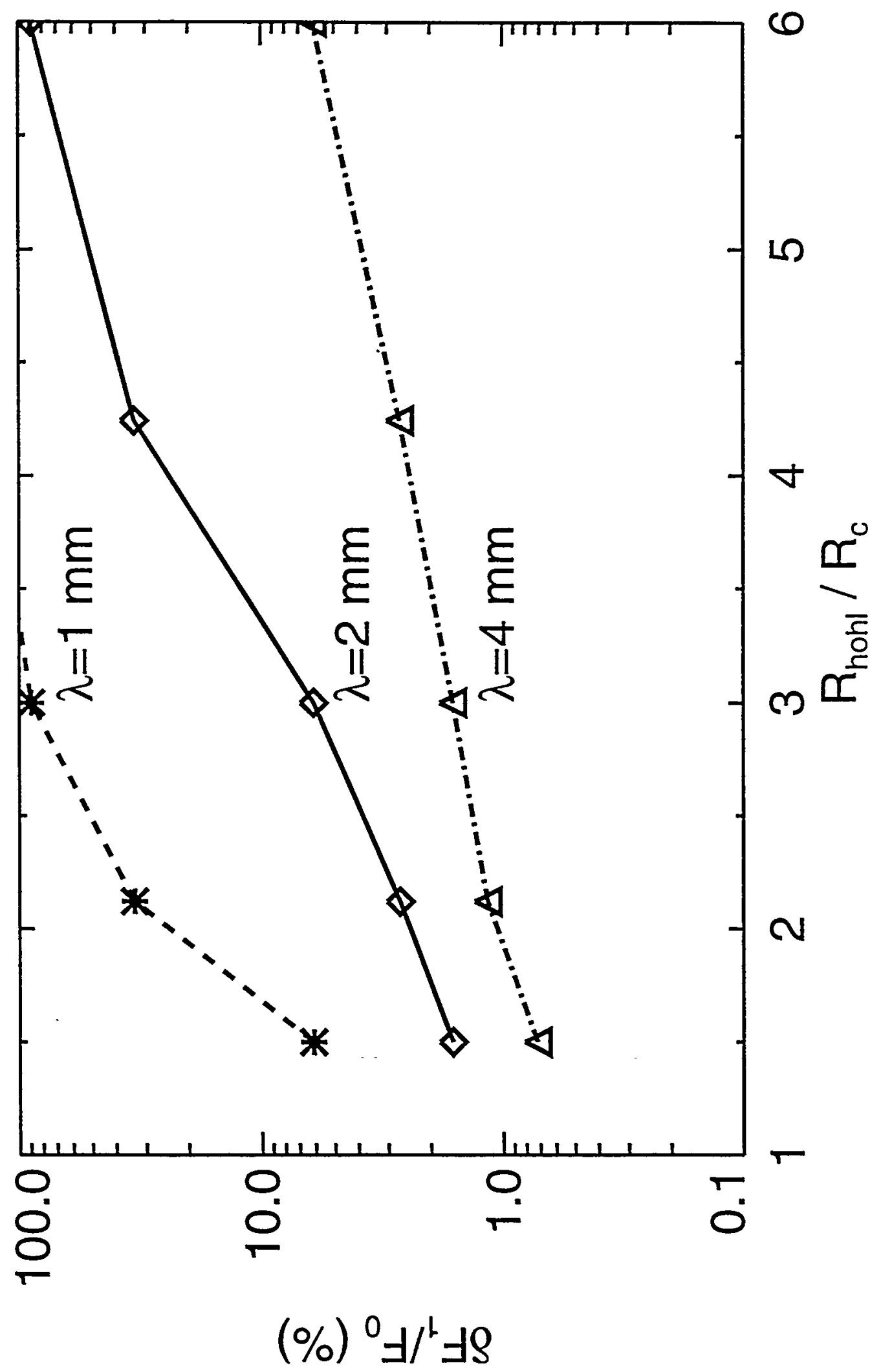




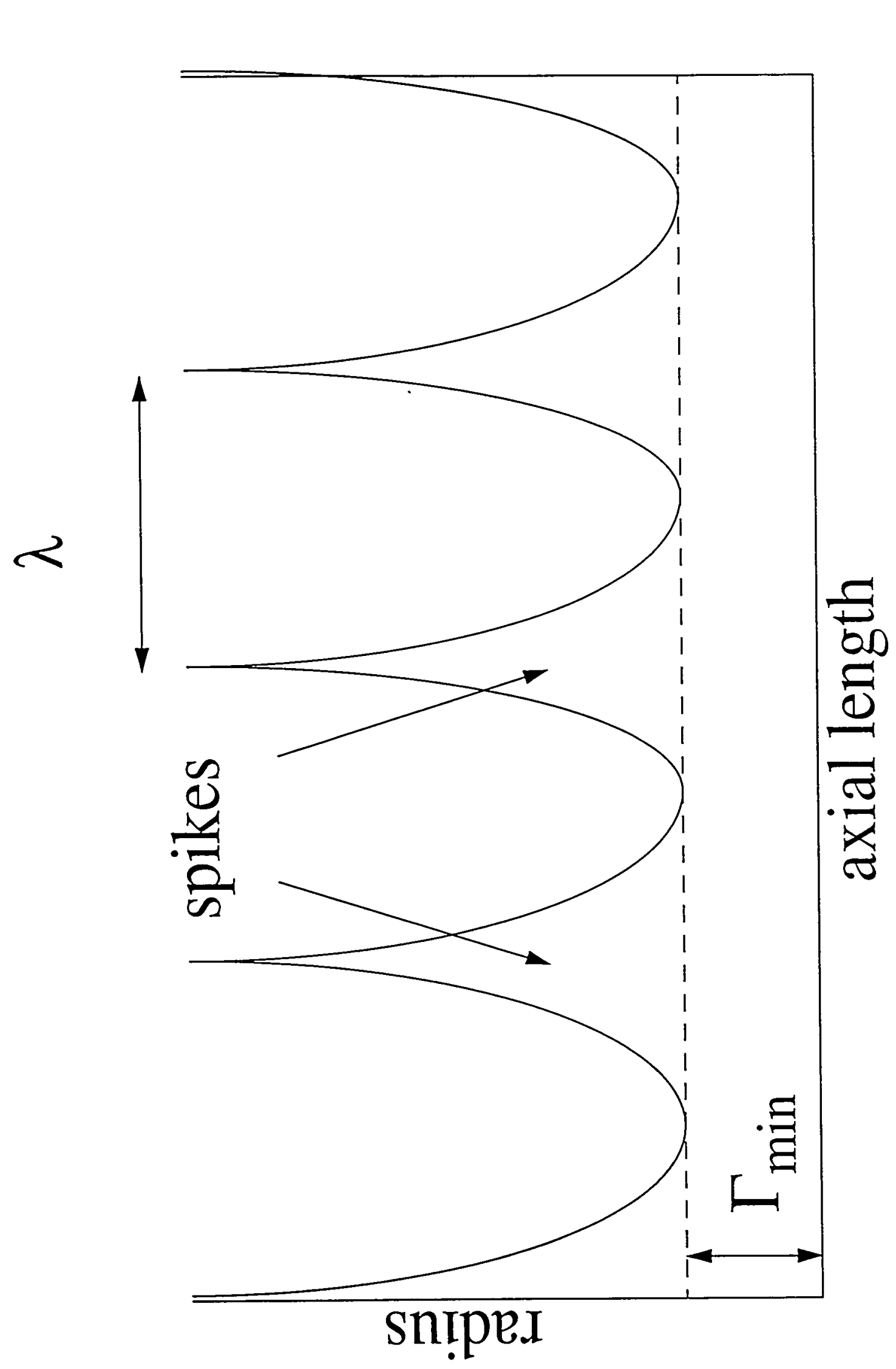




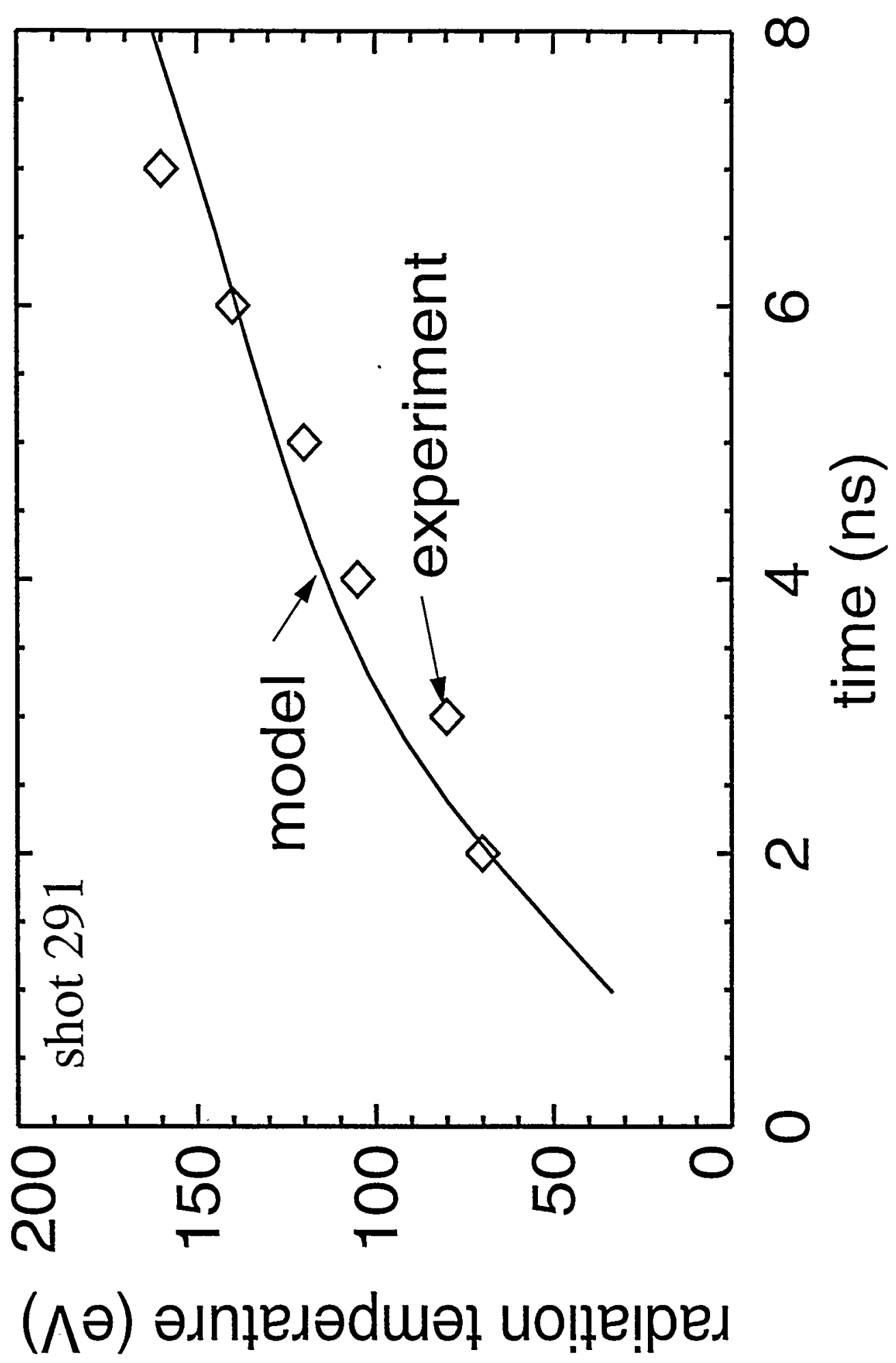




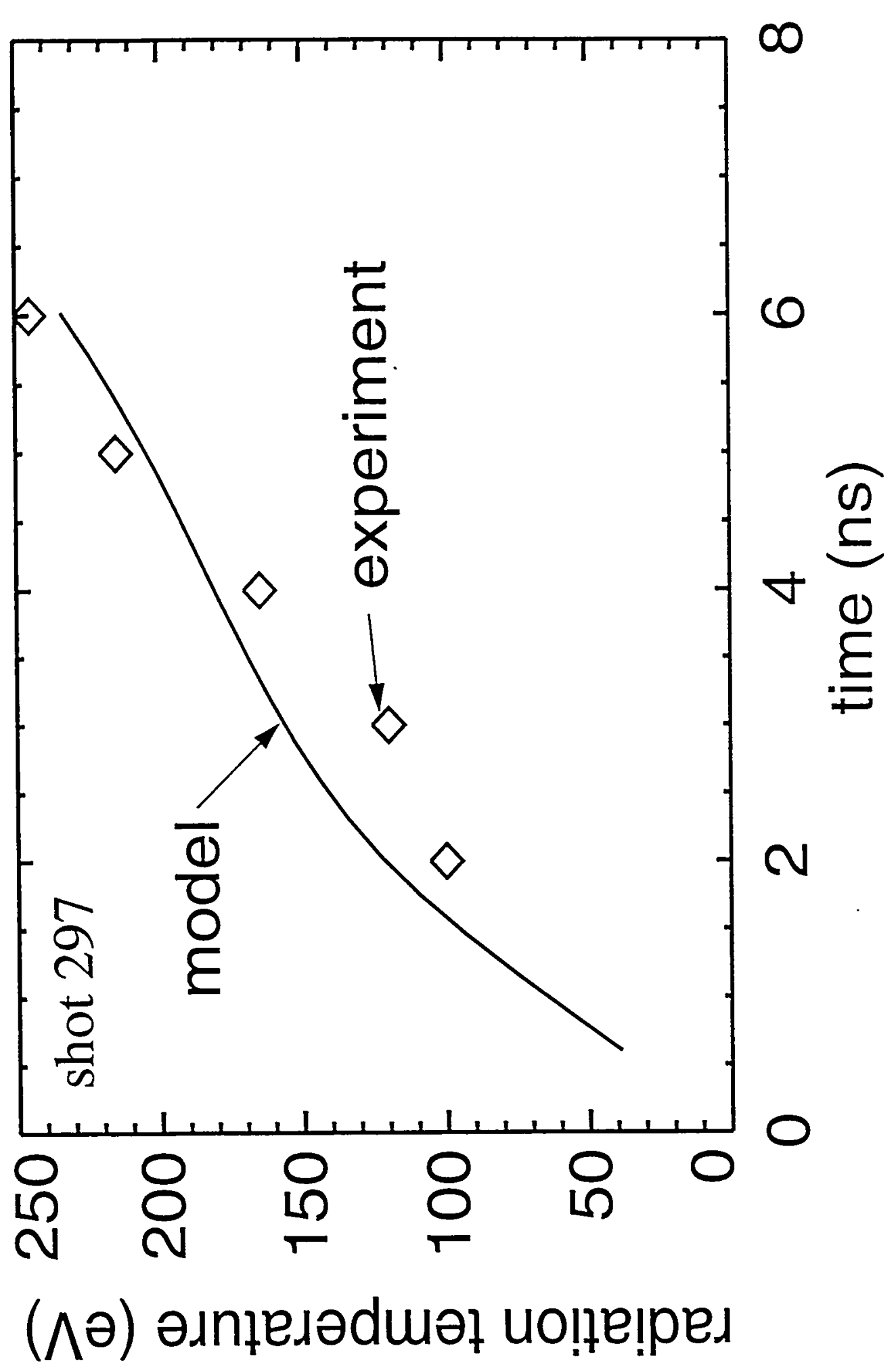




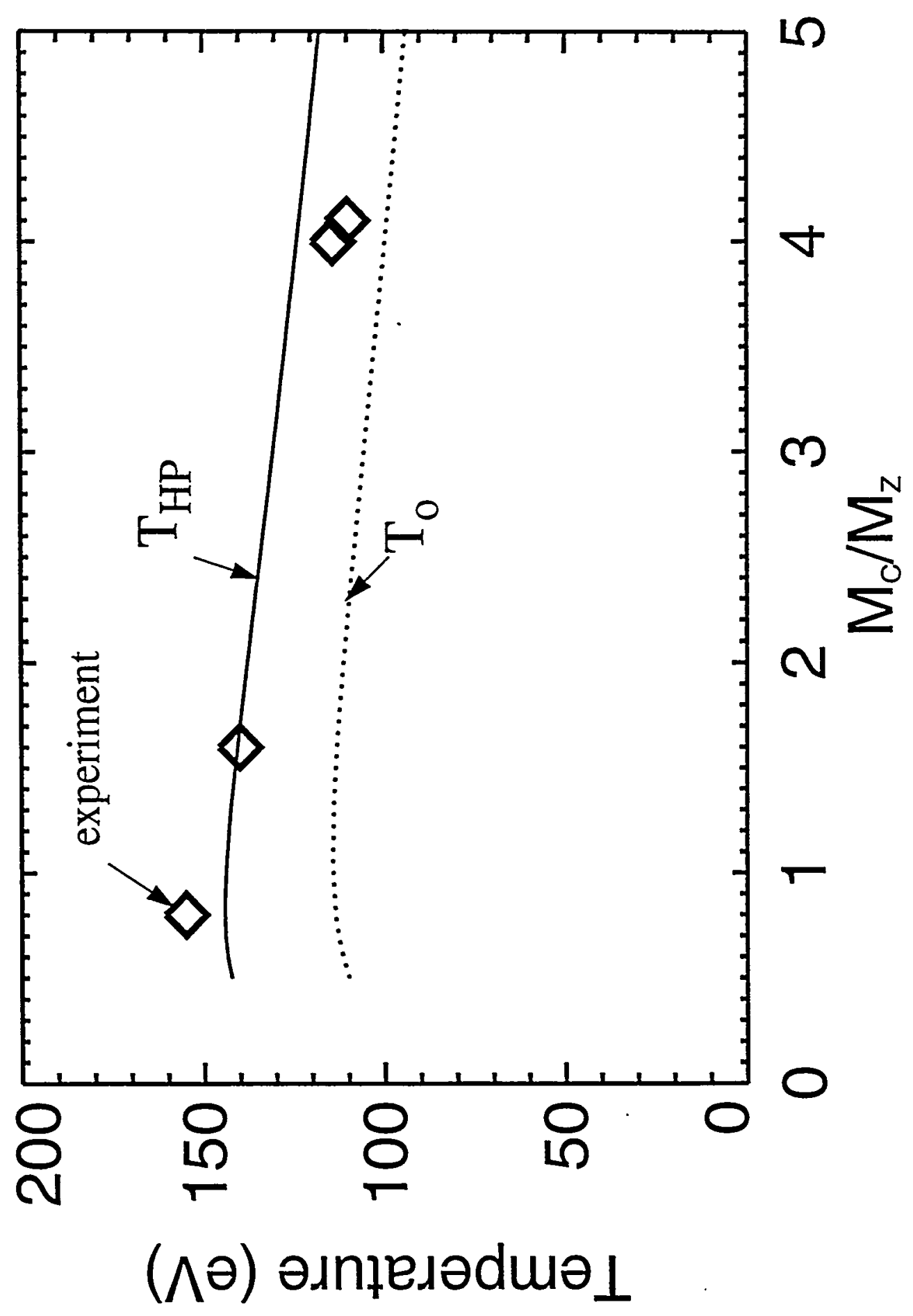




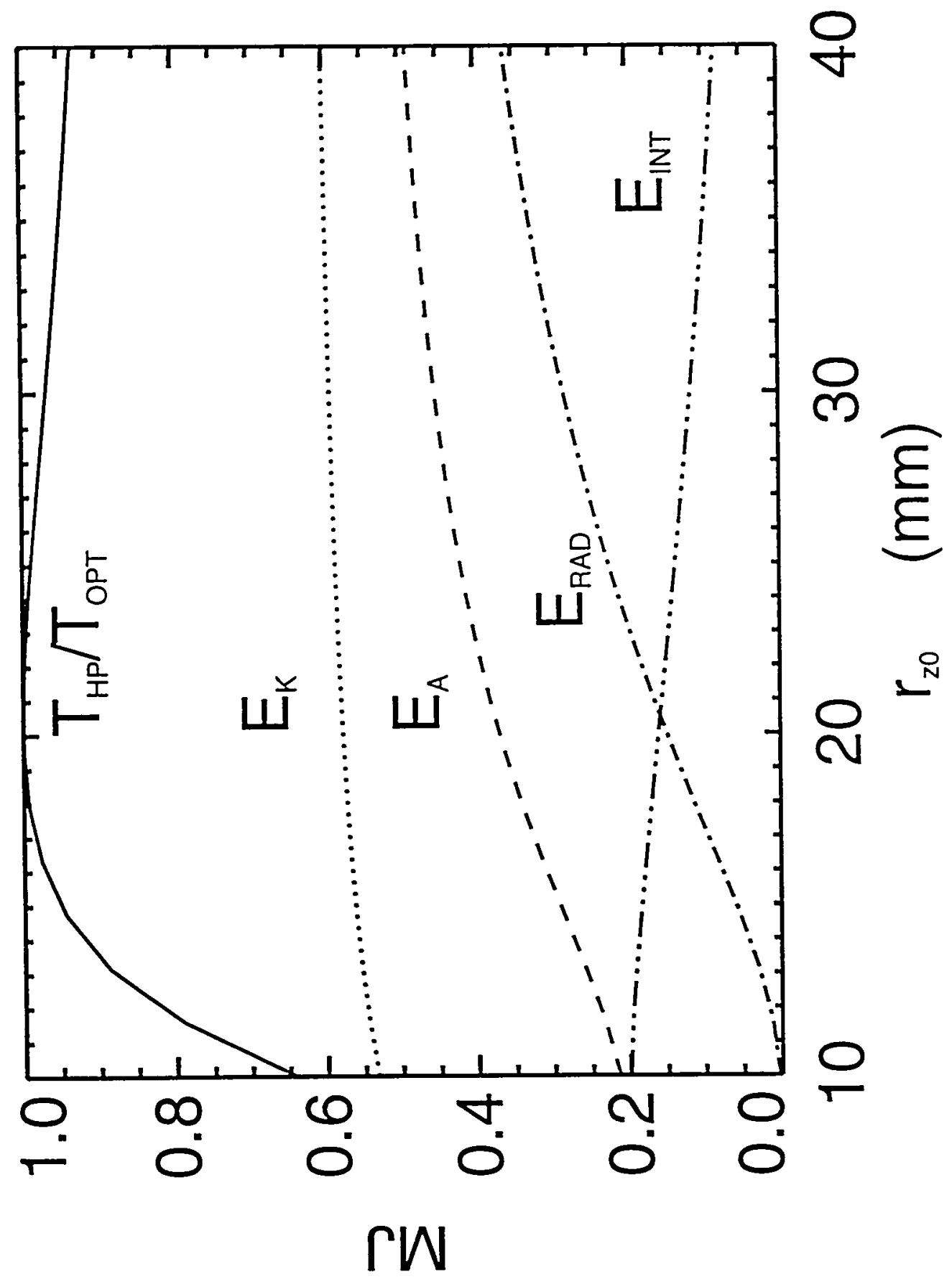




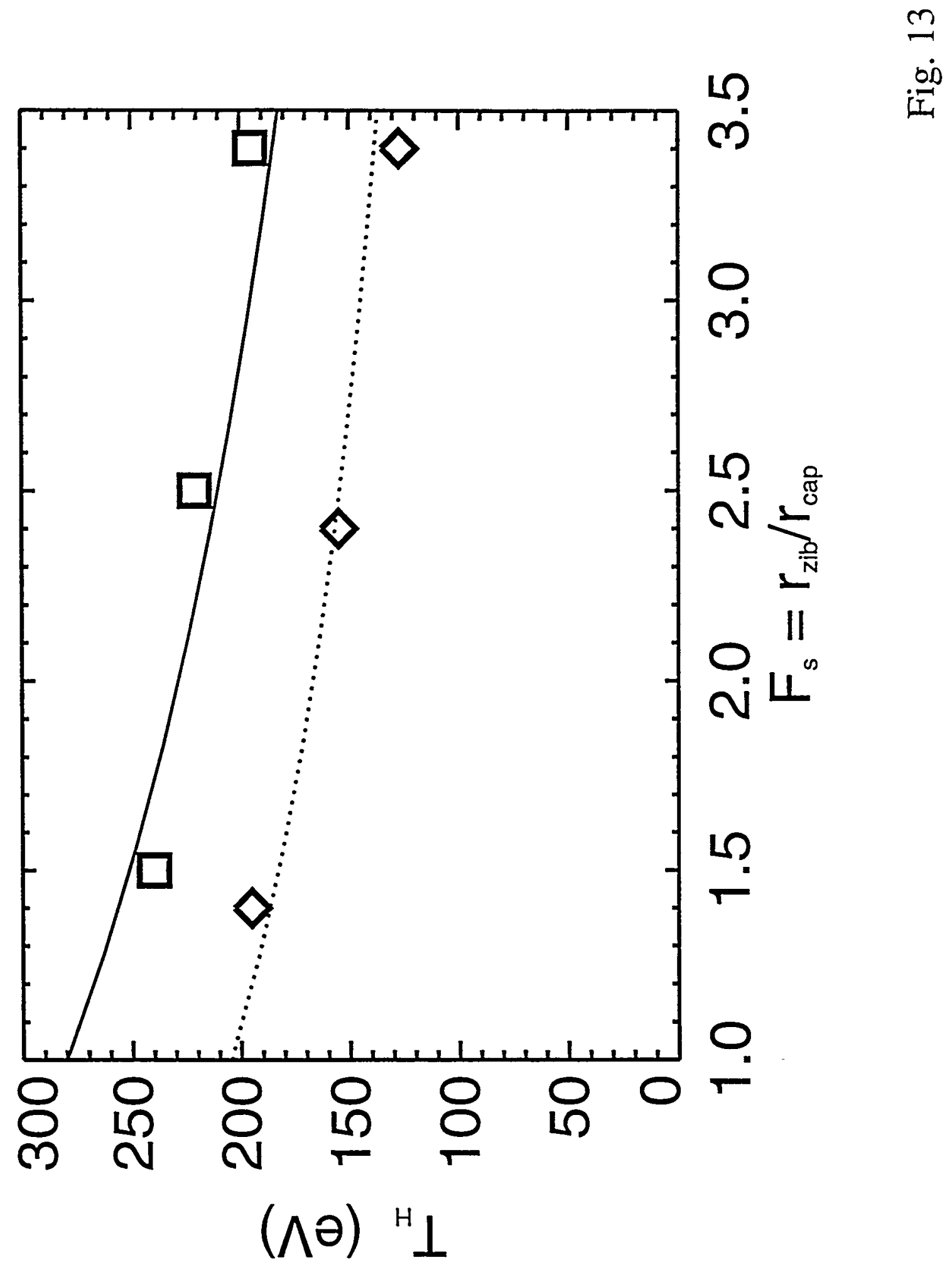



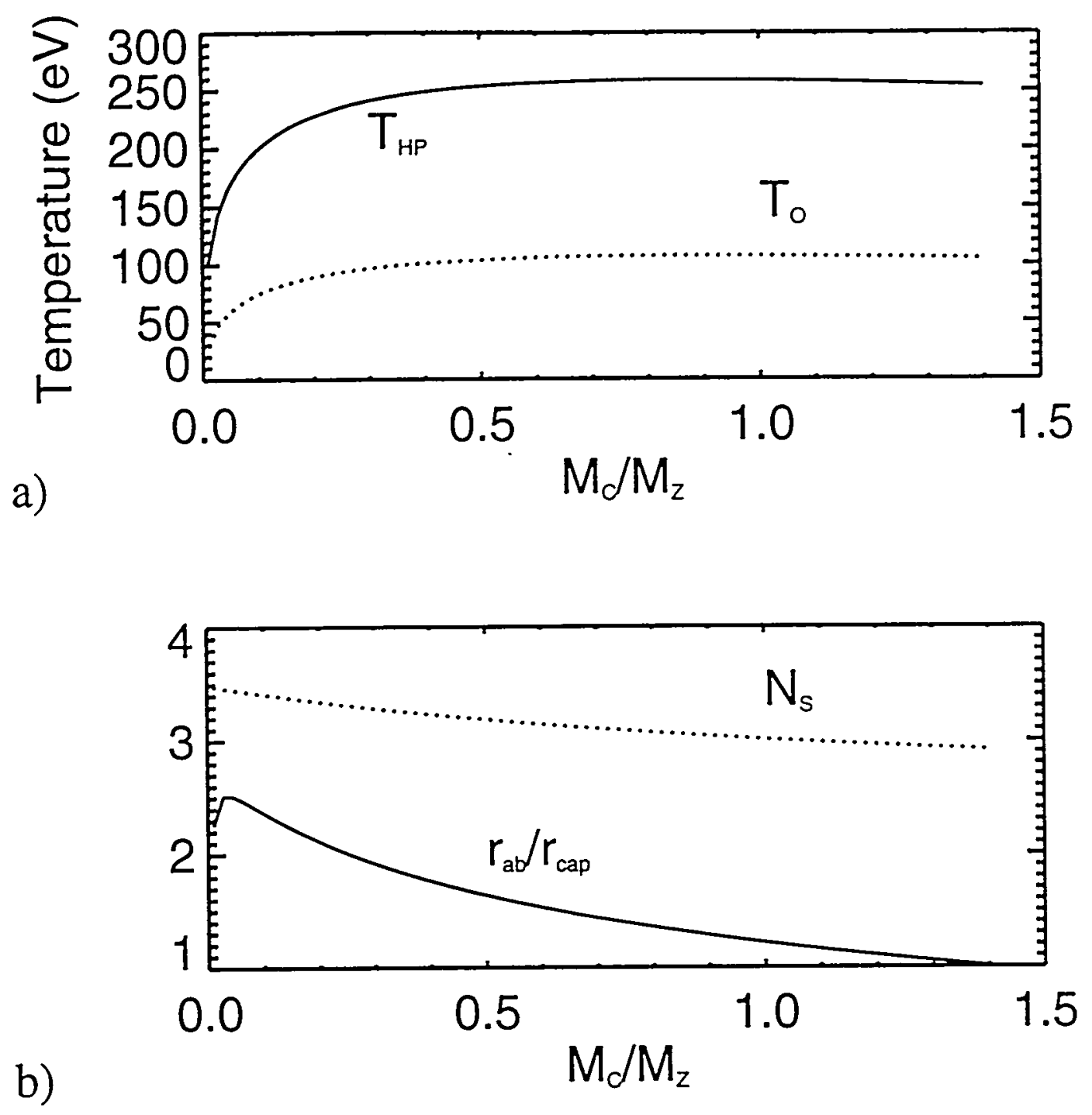

b)

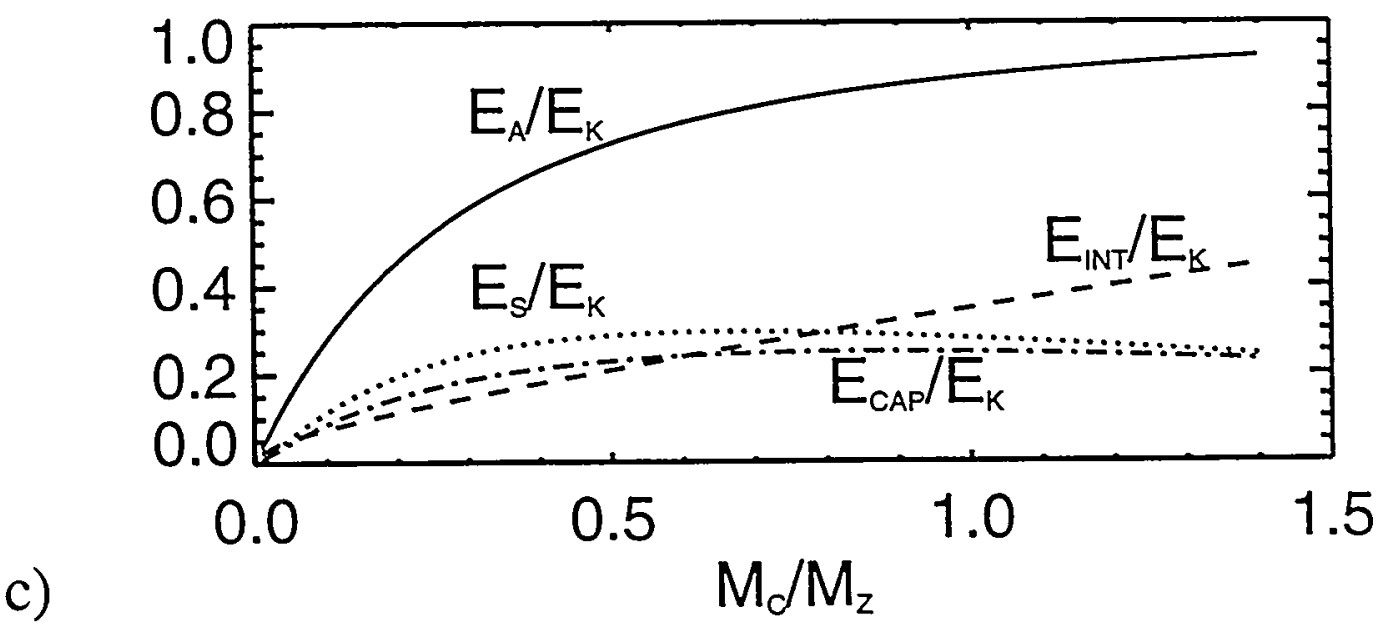

Fig. 14 
a)

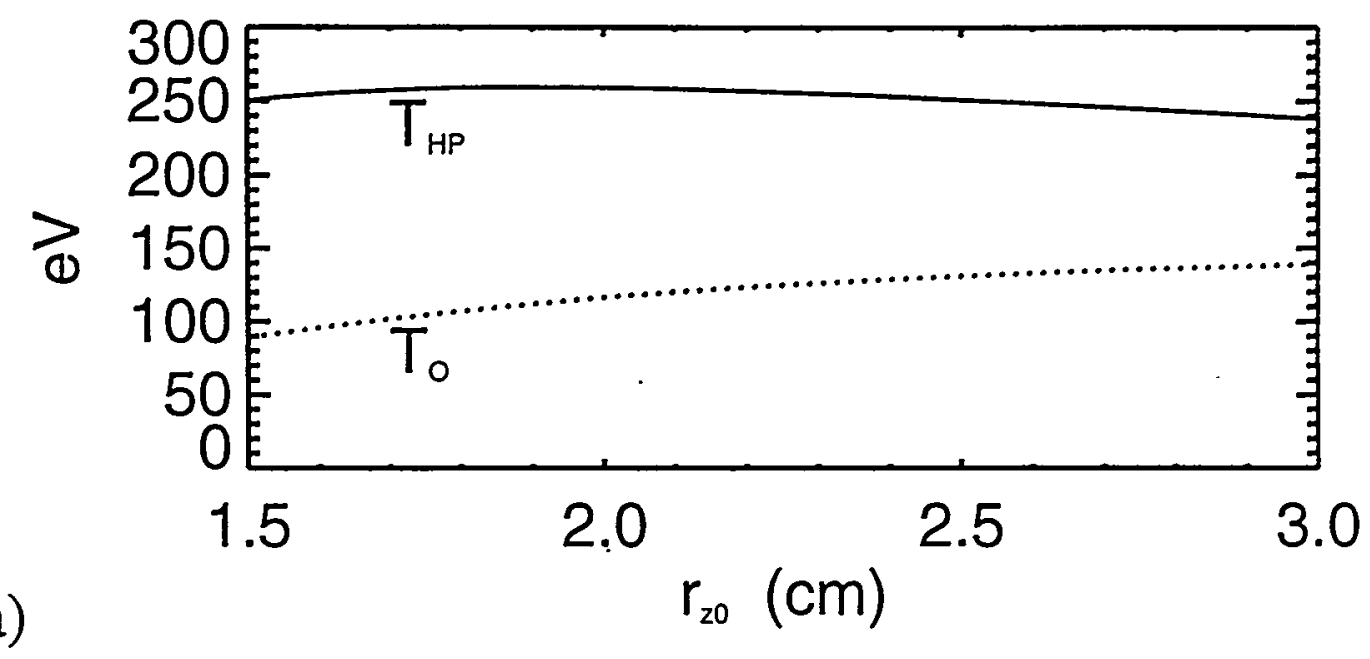

b)

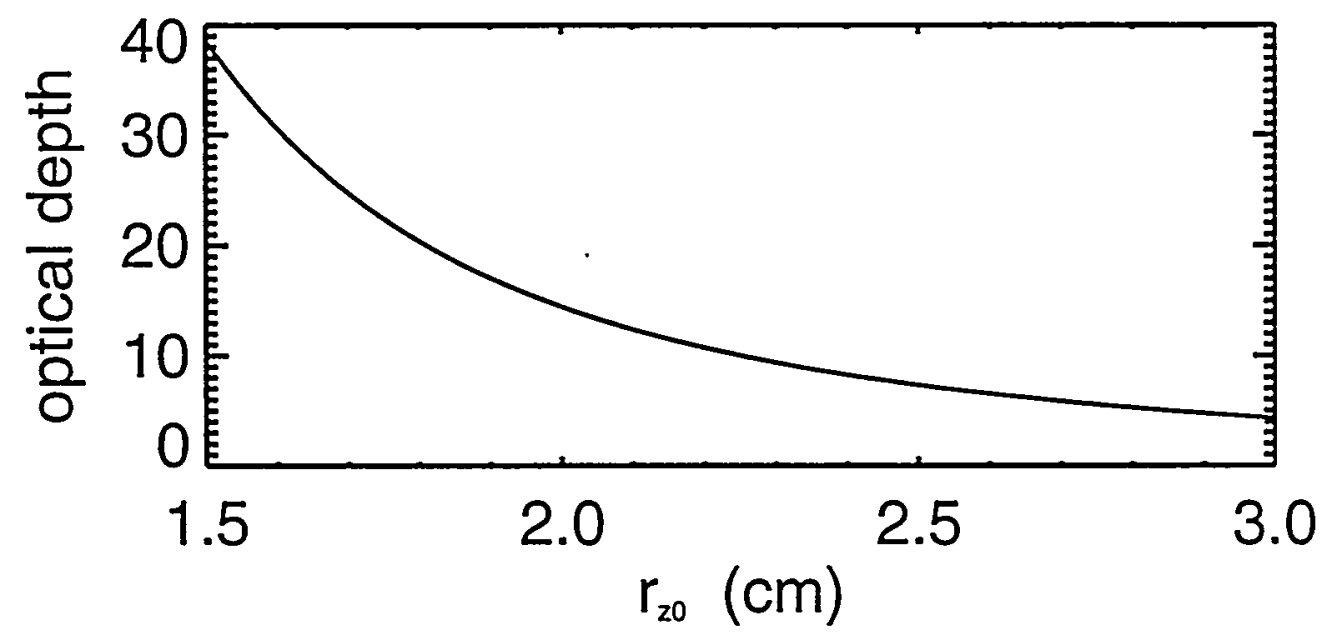

c)

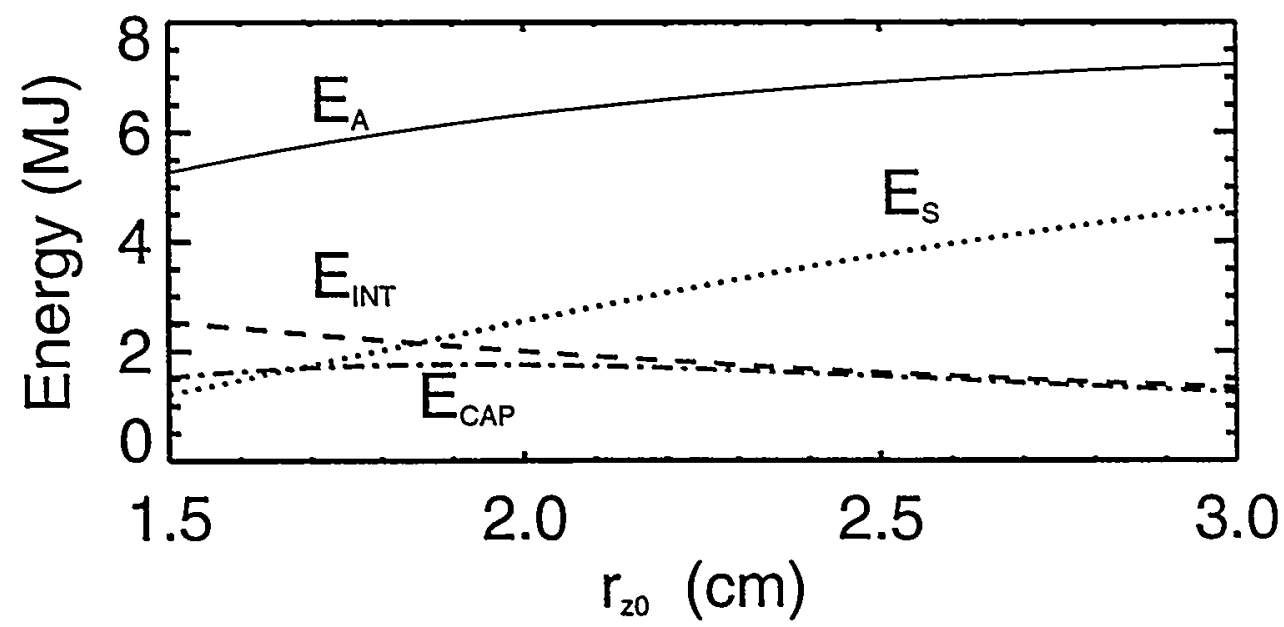

Fig. 15 

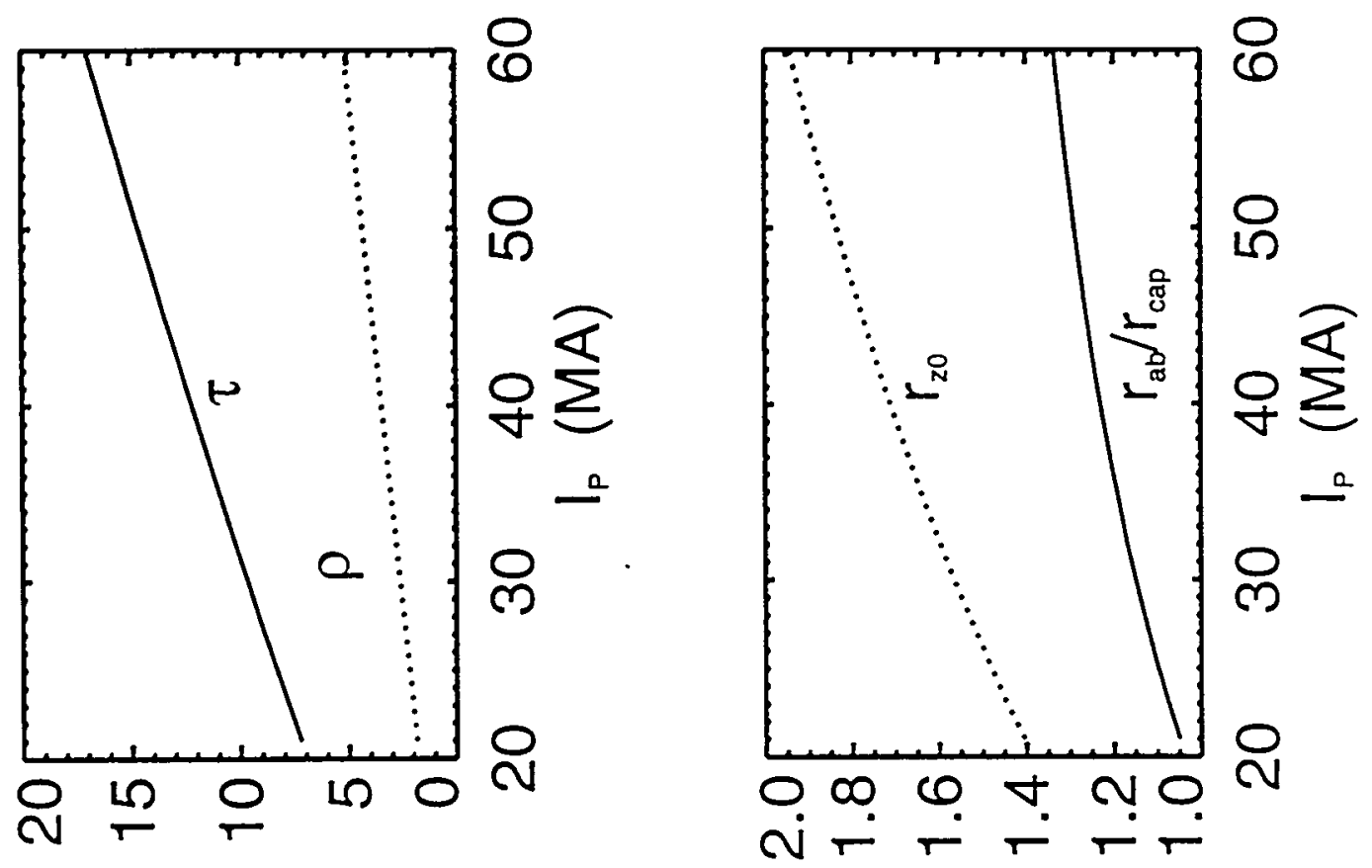

$\frac{6}{i \dot{L D}}$

L

$0 \sum$

$0^{-}$

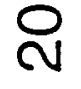

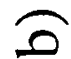

8
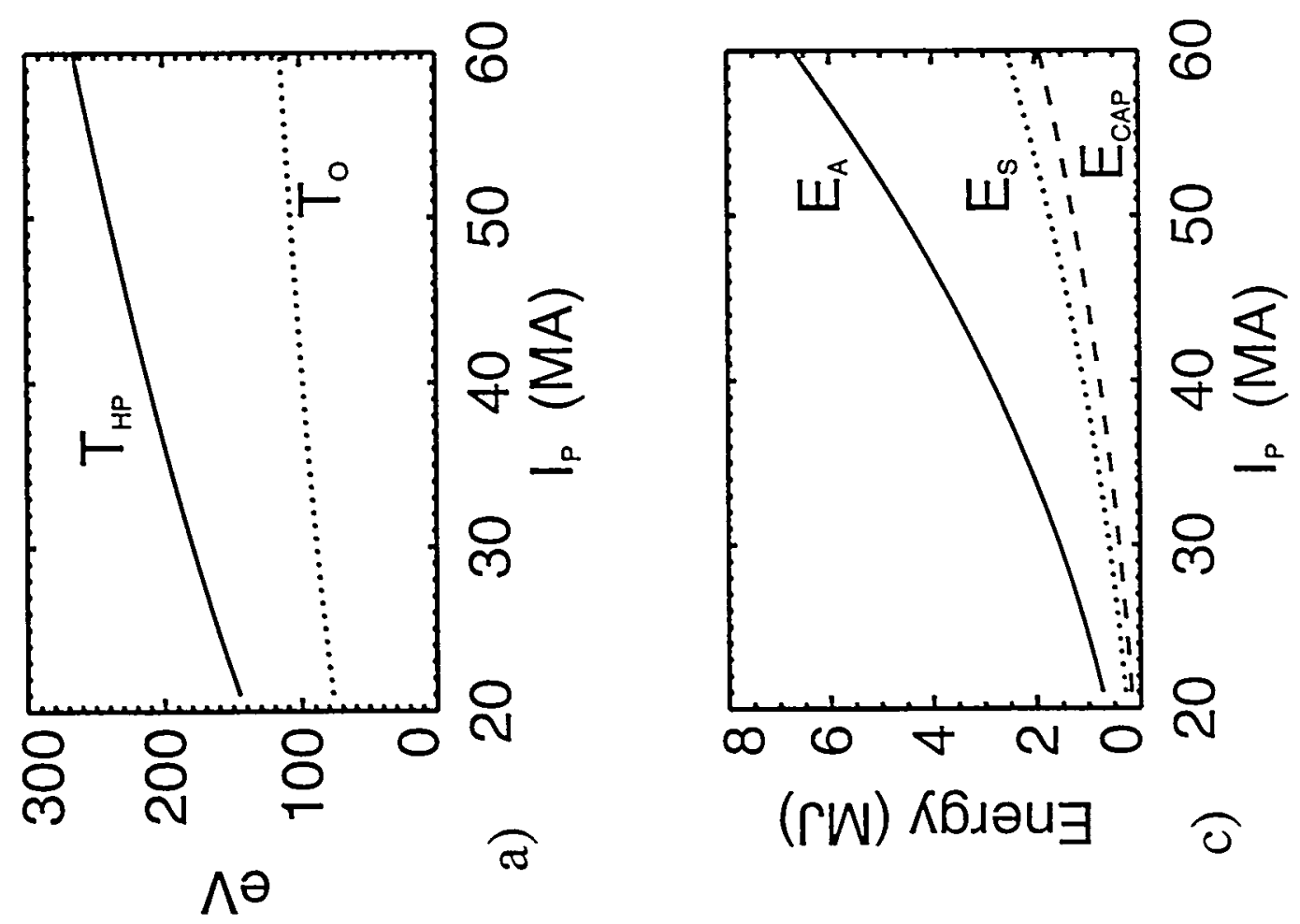

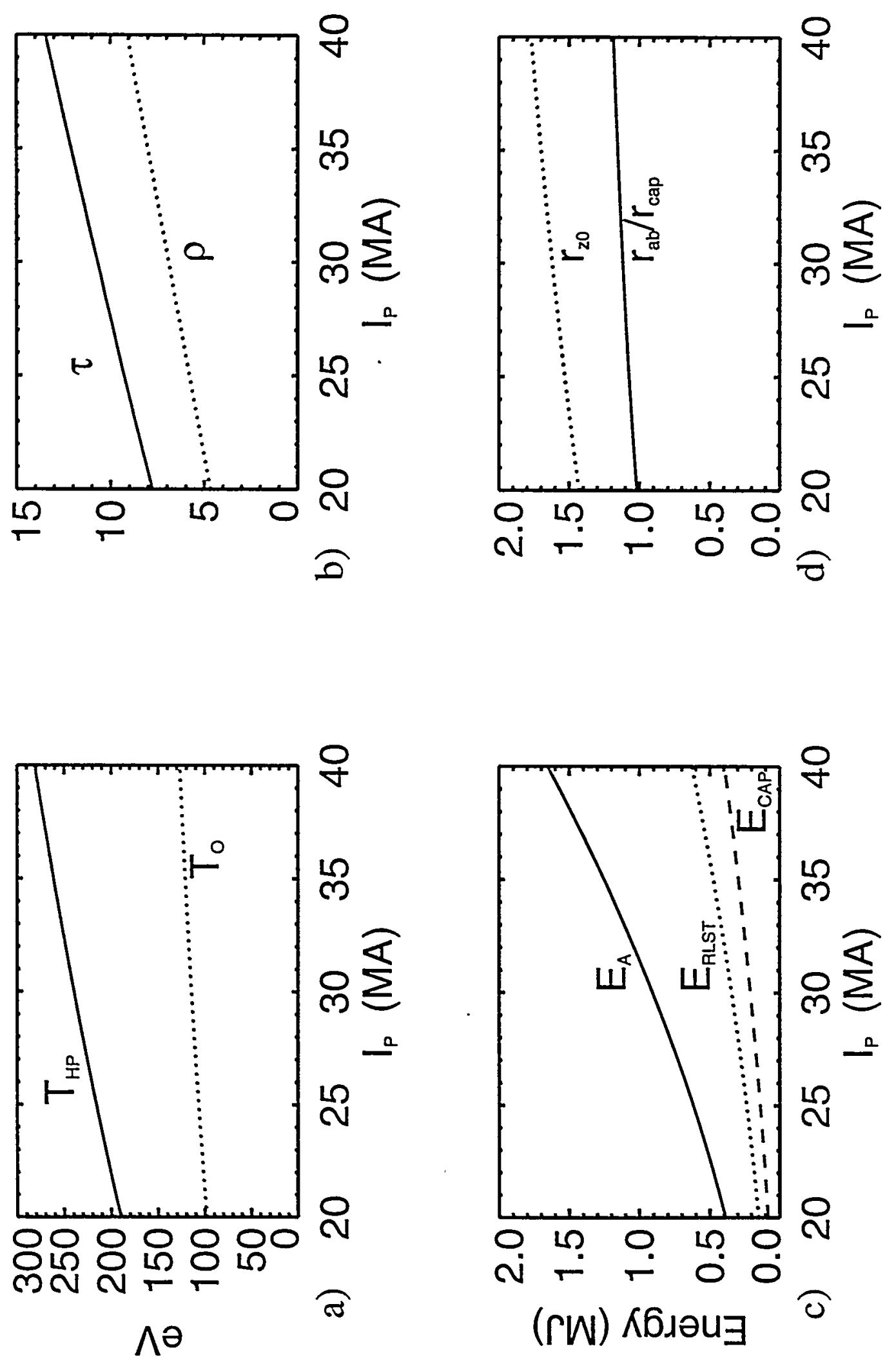


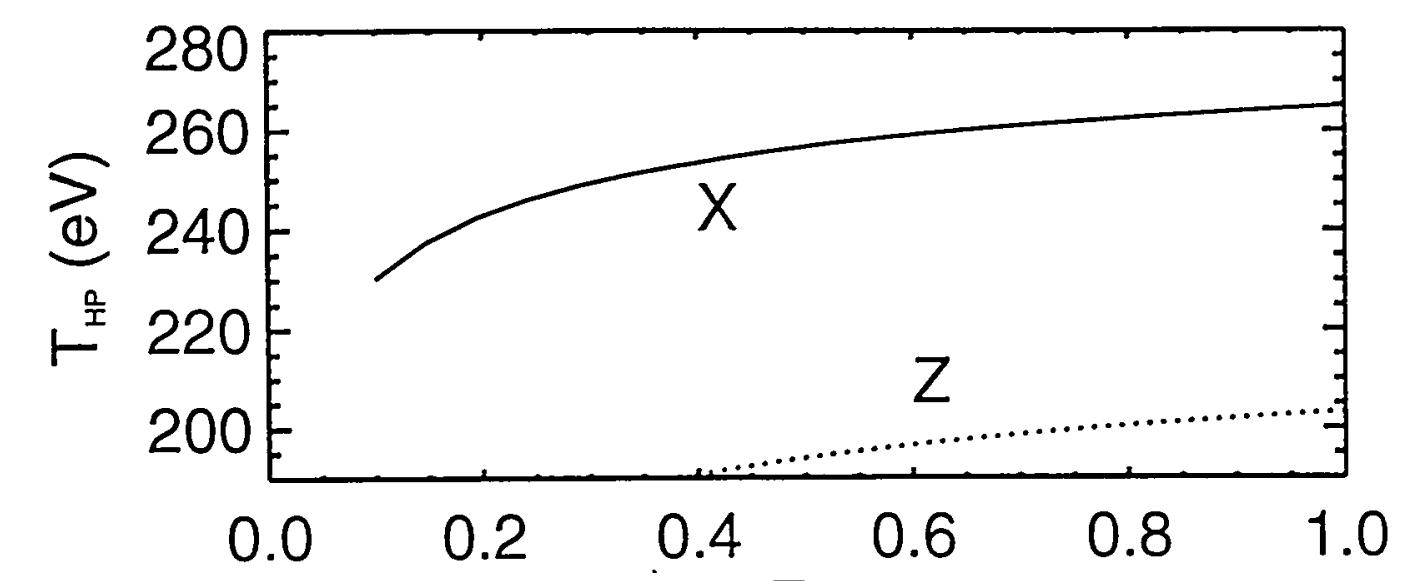

a)

$F_{\text {op }}$

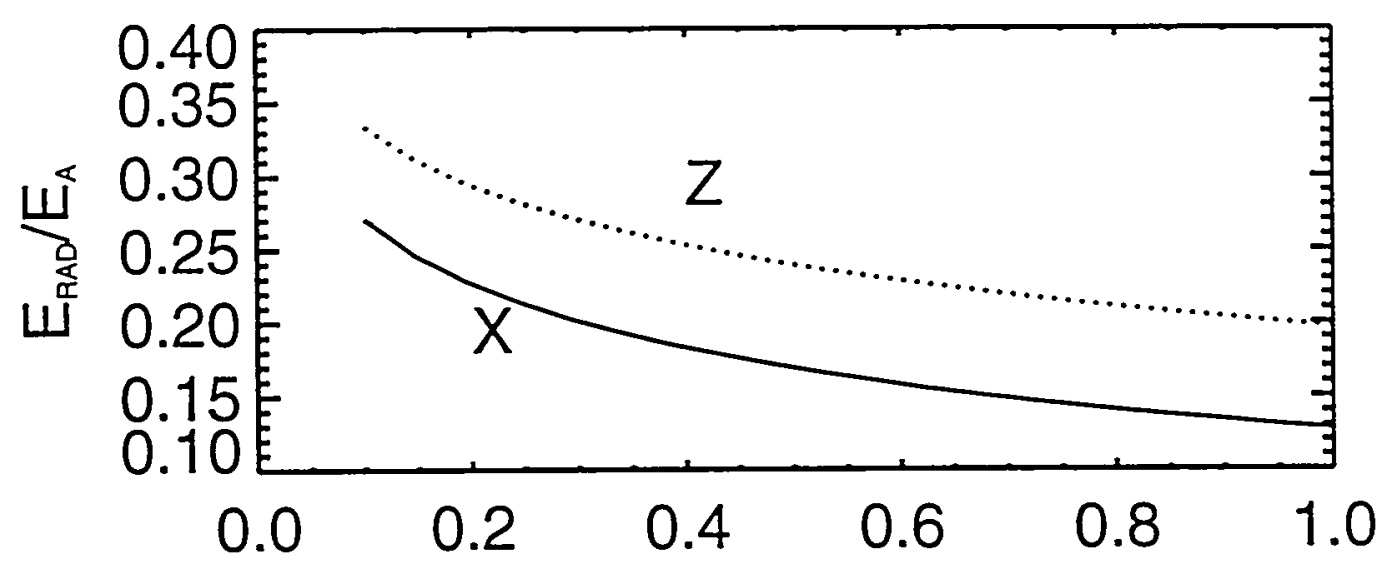

b) $F_{\text {op }}$

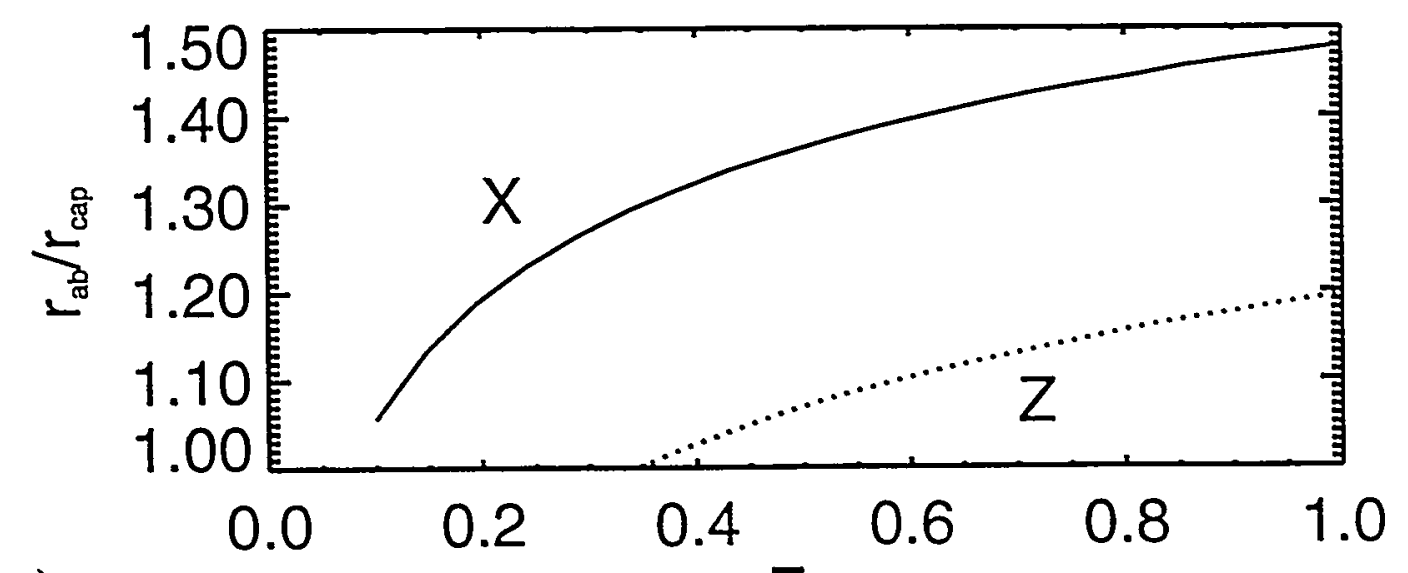

c)

$\mathrm{F}_{\mathrm{OP}}$

Fig. 18 

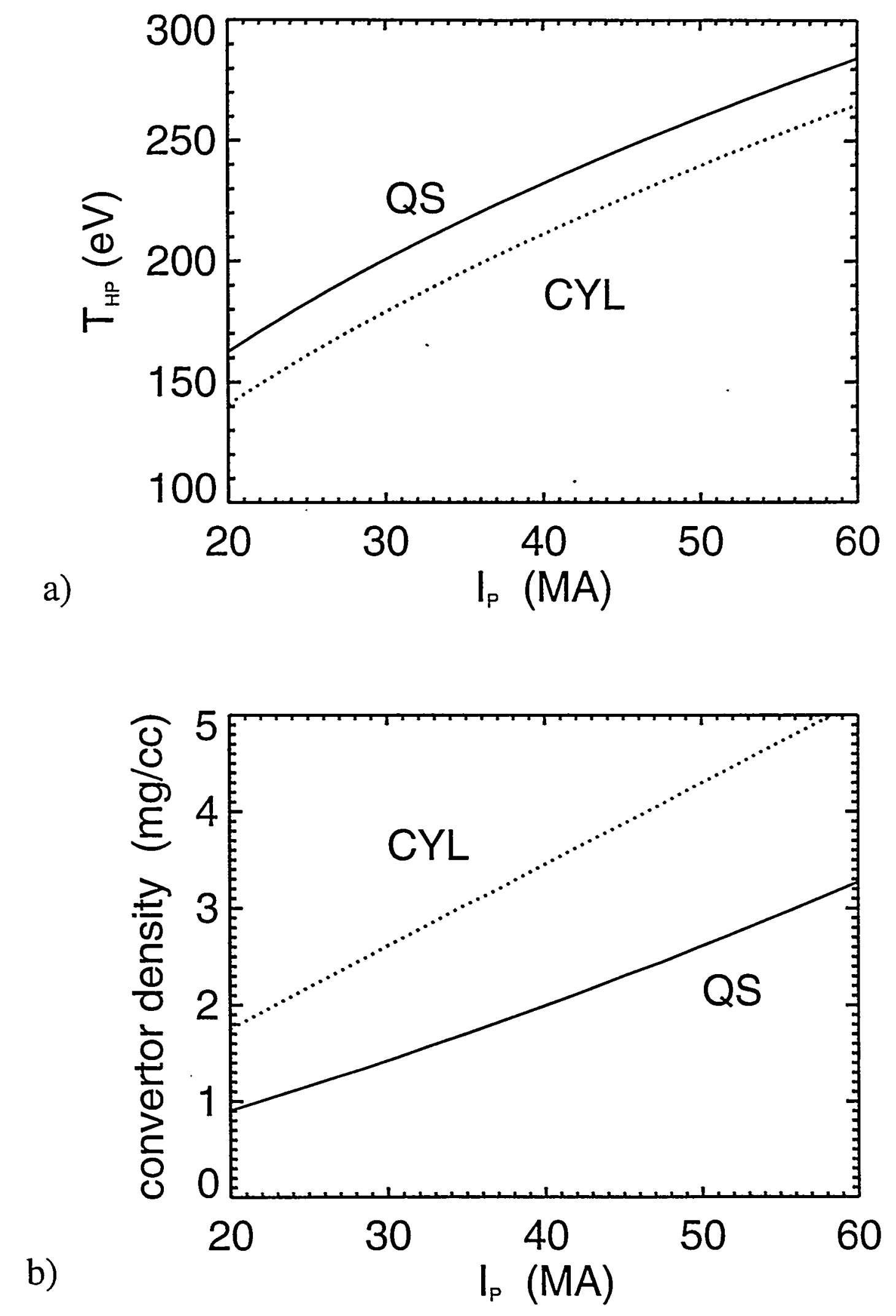

Fig. 19 


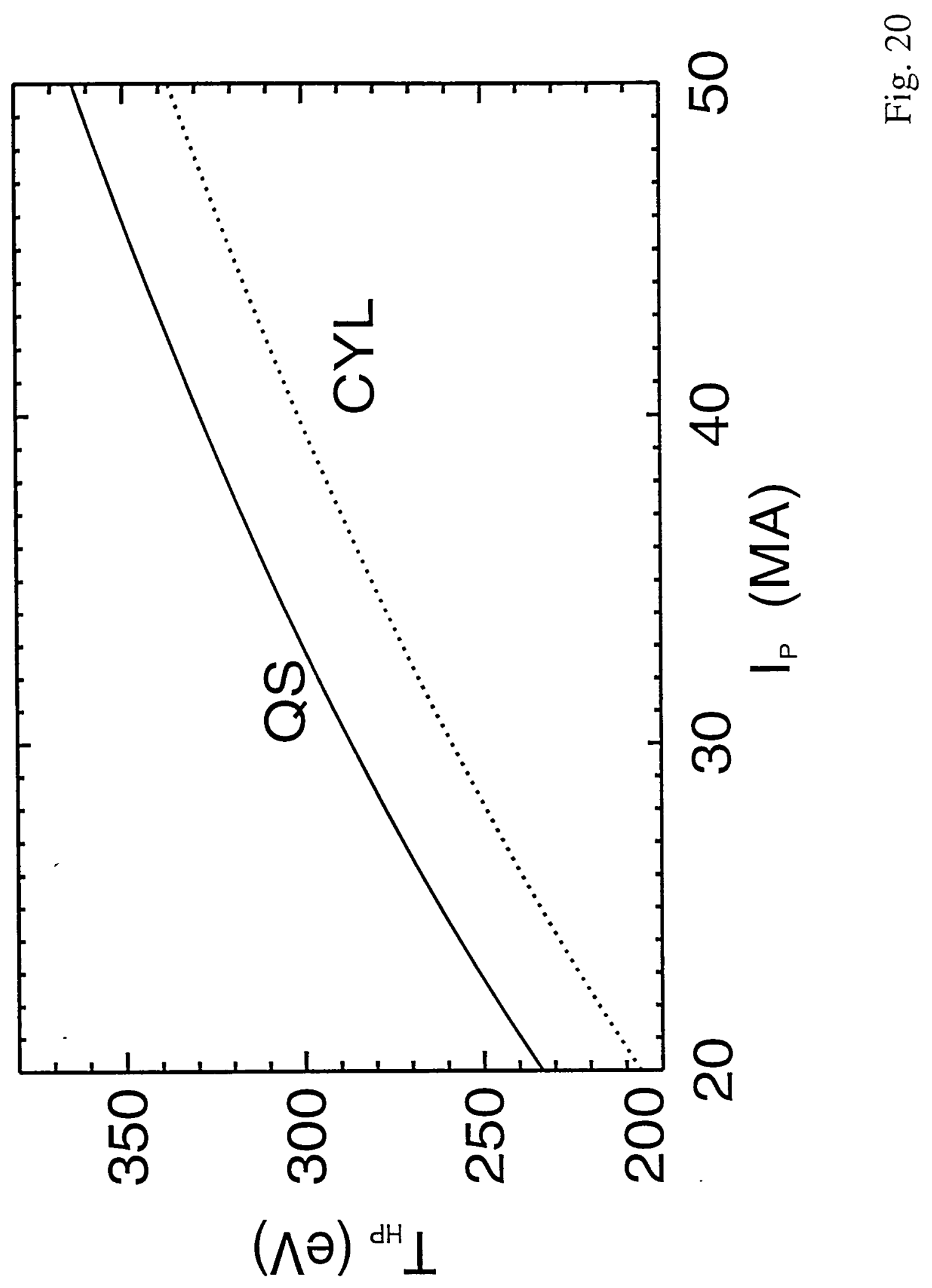




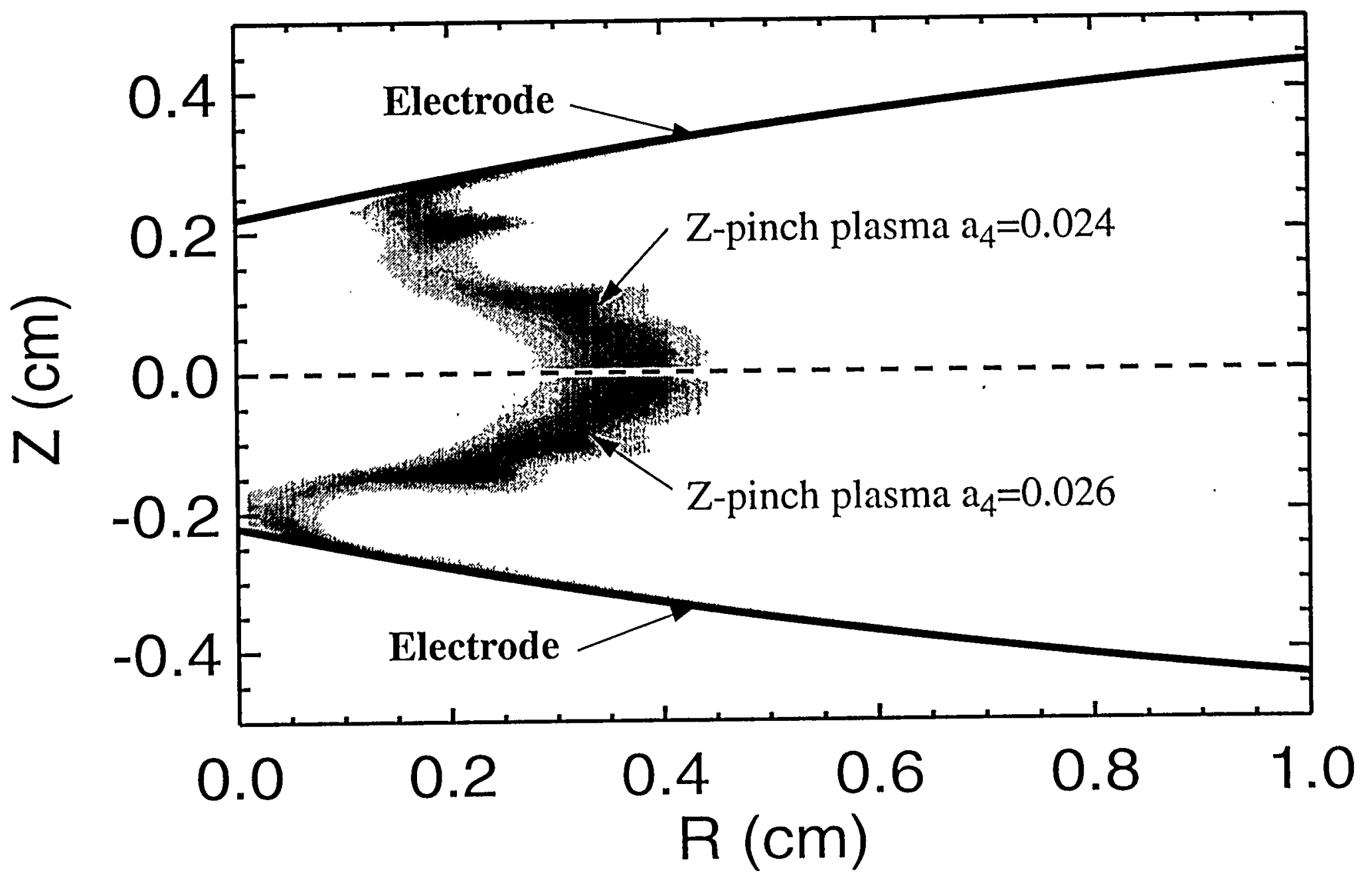




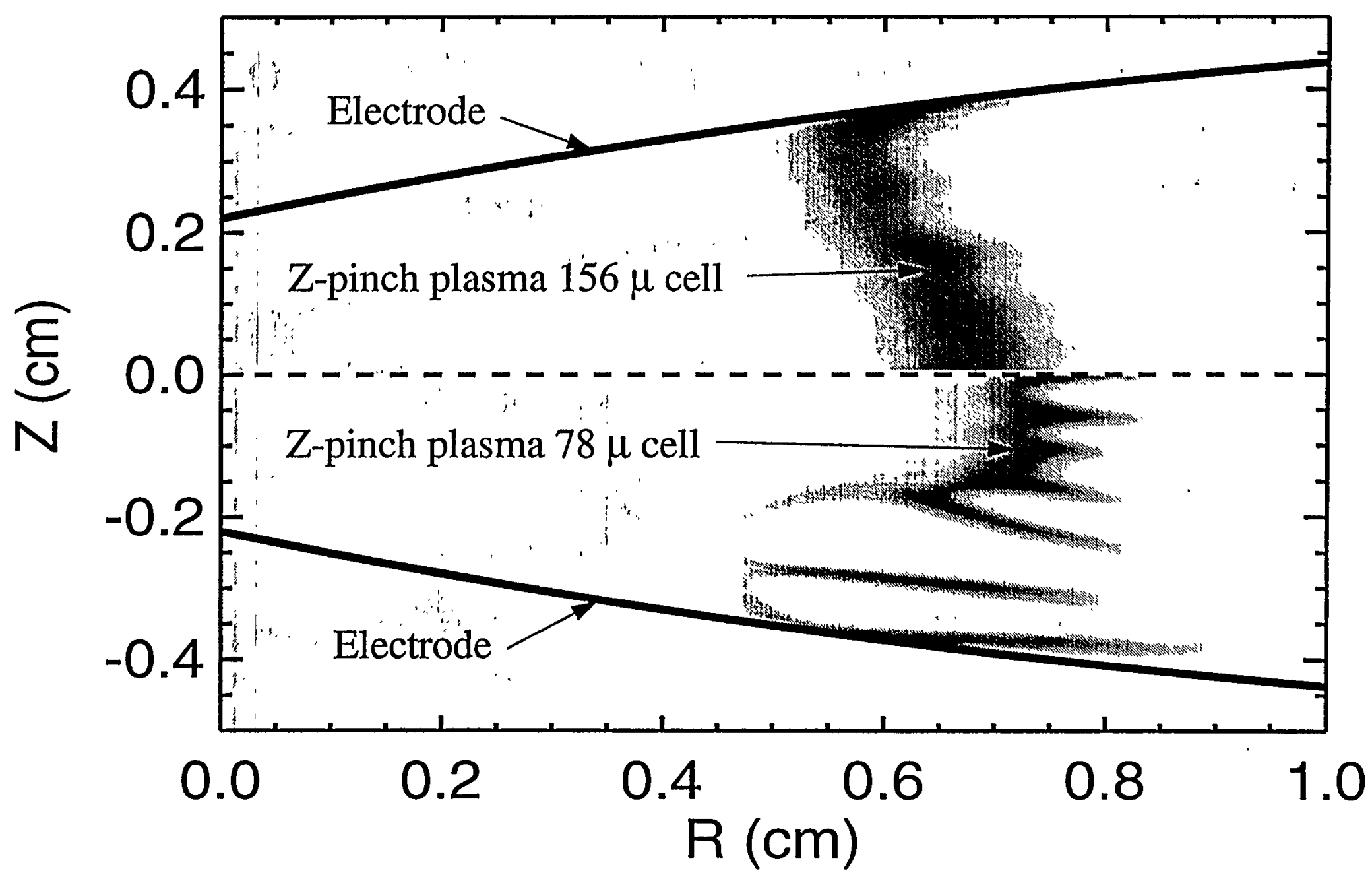

